\title{
The rotation curves shapes of late-type dwarf galaxies
}

\author{
R. A. Swaters ${ }^{1,2, \star}$, R. Sancisi ${ }^{3,4}$, T. S. van Albada ${ }^{3}$, and J. M. van der Hulst ${ }^{3}$
}

\author{
1 Department of Physics and Astronomy, Johns Hopkins University, 3400 N. Charles Str., Baltimore, MD 21218, USA \\ 2 Space Telescope Science Institute, 3700 San Martin Dr., Baltimore, MD 21218, USA \\ 3 Kapteyn Astronomical Institute, PO Box 800, 9700 AV Groningen, The Netherlands \\ 4 INAF - Osservatorio Astronomico di Bologna, via Ranzani 1, 40127 Bologna, Italy
}

Received 3 July 2008 / Accepted 25 October 2008

\section{ABSTRACT}

\begin{abstract}
We present rotation curves derived from H I observations for a sample of 62 galaxies that have been observed as part of the Westerbork H I Survey of Spiral and Irregular Galaxies (WHISP) project. These rotation curves have been derived by interactively fitting model data cubes to the observed cubes. This procedure takes the rotation curve shape, the H I distribution, the inclination, and the size of the beam into account, and makes it possible to correct for the effects of beam smearing. A comparison with higher spatial resolution $\mathrm{H} \alpha$ rotation curves available in the literature shows that there is general agreement between the two. The late-type dwarf galaxies in our sample have rotation-curve shapes that are similar to those of late-type spiral galaxies, in the sense that their rotation curves, when expressed in units of disk scale lengths, rise as steeply in the inner parts and start to flatten at two disk scale lengths. None of the galaxies in our sample have solid-body rotation curves that extend beyond three disk scale lengths. The logarithmic slopes between two disk scale lengths and the last measured point on the rotation curve is similar between late-type dwarf and spiral galaxies. Thus, whether the flat part of the rotation curve is reached or not seems to depend more on the extent of the rotation curve than on its amplitude. We also find that the outer rotation curve shape does not strongly depend on luminosity, at least for galaxies fainter than $M_{R} \sim-19$. We find that in spiral galaxies and even in the central regions of late-type dwarf galaxies, the shape of the central distribution of light and the inner rise of the rotation curve are related. This implies that galaxies with stronger central concentrations of light also have higher central mass densities, and it suggests that the luminous mass dominates the gravitational potential in the central regions, even in low surface brightness dwarf galaxies.
\end{abstract}

Key words. surveys - galaxies: dwarf - galaxies: irregular - galaxies: kinematics and dynamics

\section{Introduction}

The shape and amplitude of rotation curves are directly related to the gravitational potential in the midplane of the galaxy. Hence, a relation is expected between the main mass components in a galaxy and the shape of the rotation curve. If the luminous mass density is dynamically significant, the rotation curve shape will be related to the distribution of light. On the other hand, dark matter may weaken such a relation, in particular if the dark matter dominates the potential. Therefore, in principle, a comparison between rotation curves shapes and the distribution of luminous mass can provide insight in the amount and distribution of dark matter in galaxies, and it can indicate how the structural properties of the dark and luminous components are linked. Moreover, because this link is probably the result of the process of galaxy formation, studying this link between dark and luminous matter is relevant for theories of galaxy formation.

The relation between the luminous properties of galaxies and their rotation curves has been a topic of study for many years (e.g., Rubin et al. 1985; Kent 1986, 1987; Corradi \& Capaccioli 1990; Persic et al. 1996; Rhee 1996; Verheijen 1997; Catinella et al. 2006). These studies, however, are mainly based on high surface brightness spiral galaxies with absolute magnitudes brighter than $M_{B} \sim-18$. To better understand the relation between luminous and dark mass and its implications for

^ Present address: Department of Astronomy, University of Maryland, College Park, MD 20742-2421, USA.

e-mail: swaters@astro.umd.edu the structure and formation of galaxies it is necessary to include galaxies with lower luminosities and lower surface brightnesses.

In recent years, considerable effort has been invested in studies of rotation curve shapes of dwarf and low surface brightness galaxies (e.g., de Blok et al. 1996; de Blok \& McGaugh 1997; Stil 1999; Swaters 1999; Swaters et al. 2000; van den Bosch et al. 2001; van den Bosch \& Swaters 2001; de Blok et al. 2001; Marchesini et al. 2002; de Blok \& Bosma 2002, herafter dBB; Swaters et al. 2003a, hereafter SMvdBB; Rhee et al. 2004; Simon et al. 2005; Spekkens et al. 2005; Kuzio de Naray et al. 2006; Chengalur et al. 2008). Most of these studies focused on the shape of the rotation curve in the central regions of these galaxies, because these inner rotation curve shapes provide a powerful test of models of galaxy formation in a cold dark matter dominated universe (e.g., Navarro et al. 1996, 1997; Bullock et al. 2001; Power et al. 2003). Because high spatial resolution is necessary to determine the inner rotation curve shapes, these studies of dwarf and low surface brightness galaxies have mostly relied on optical spectroscopy of the $\mathrm{H} \alpha$ emission line. Unfortunately, the $\mathrm{H} \alpha$ emission is usually only detected in the optical disk. Hence, most of these rotation curves do not extend to large enough radii to measure the rotation curve shape outside the optical disk. Instead, HI rotation curves are needed for that purpose.

To date, relatively little work has been done to investigate the relations between luminous properties and the H I rotation curve shape in the dwarf galaxy regime. The studies by Tully et al. (1978), Broeils (1992a) and Côté et al. (2000), which 
each comprised a handful of dwarf galaxies, led the picture that late-type dwarf galaxies have low amplitude, slowly rising rotation curves that keep rising to the last measured point. Thus, the rotation curves shapes of dwarf galaxies seemed different from those of spiral galaxies, which, in general, have a steep rise in the inner regions, followed by relatively flat outer parts (e.g., Bosma 1978, 1981a,b; Begeman 1987, 1989). This was confirmed and refined by Casertano \& van Gorkom (1991, hereafter CvG) and Broeils (1992a). They investigated the correlations between the outer slopes of the rotation curves and the maximum rotation velocities, and found that the rotation curves, when expressed in units of the optical radius, rise more slowly towards lower rotation amplitudes.

To further our understanding of the relation between the distribution of light and the shape of the rotation curves for dwarf galaxies, a large sample with well-defined rotation curves and optical properties is needed. Such a sample is presented in Swaters et al. (2002, hereafter Paper I); it contains 73 late-type dwarf galaxies. For these galaxies optical properties have been presented in Swaters \& Balcells (2002, hereafter Paper II). This sample forms the basis of the study of the rotation curves of late-type dwarf galaxies presented here (see also Swaters 1999), and of studies of their dark matter properties published earlier (Swaters 1999; van den Bosch \& Swaters 2001; SMvdBB).

The structure of this paper is as follows. In Sect. 2 we describe the sample selection. Section 3 describes in detail the procedure used to derive the rotation curves. We give a brief description of a number of individual galaxies in Sect. 4 , and in Sect. 5 we compare our $\mathrm{H}$ I rotation curves with long-slit $\mathrm{H} \alpha$ rotation curves available in the literature. The relations between the rotation curve shapes and the luminous properties are discussed in Sect. 6. In Sect. 7 we discuss the results and present our conclusions. In Appendix A we present the data in graphical form.

\section{The sample}

The galaxies in this sample have been observed as part of the WHISP project (Westerbork H I Survey of Spiral and Irregular Galaxies) which aimed at mapping several hundred nearby spiral and irregular galaxies in H I with the WSRT. The WHISP sample was selected from the Uppsala General Catalogue of Galaxies (UGC, Nilson 1973), taking all galaxies with declinations north of $20^{\circ}$, blue major axis diameters larger than $1.5^{\prime}$ and measured H I flux densities larger than $100 \mathrm{mJy}$. For the present purpose, we defined as dwarf galaxies all galaxies with Hubble types later than Sd, and spiral galaxies of earlier Hubble types for which the absolute $B$-band magnitudes are fainter than -17 . For a detailed description of the selection criteria and the general goals of the WHISP project, see Paper I.

The H I observations on which the rotation curves presented here are based, are discussed in detail in Paper I. The typical full resolution of the observations is $14^{\prime \prime} \times 14^{\prime \prime} / \sin \delta$. In general the signal-to-noise ratios at this resolution are low, and therefore $\mathrm{H}$ I maps and velocity fields are given at $30^{\prime \prime}$ resolution (see Appendix A).

The sample of late-type dwarf galaxies presented here is identical to the sample of Paper I, except for four galaxies that have been omitted because they are interacting with a close companion: UGC 1249, UGC 5935, UGC 6944 and UGC 7592. The remaining sample, listed in Table 2, consists of 69 latetype dwarf galaxies. For 62 of those rotation curves have been derived.
In addition to the $\mathrm{H}$ I data, optical $R$-band data have also been obtained for these galaxies. Details of these observations and the data reduction are presented in Swaters \& Balcells (2002). The absolute magnitudes, scale lengths and surface brightnesses as derived in Paper II are listed in Table 2.

\section{The rotation curves}

\subsection{Derivation of the rotation curves}

Usually, rotation curves are derived by fitting tilted-ring models to the observed velocity fields (e.g., Begeman 1987). In principle, this method can be applied to the galaxies in this sample as well, provided that the requirements for fitting a tiltedring model are met (see Begeman 1987). However, because the galaxies in our sample are often small and the HI distribution is usually irregular and clumpy, and because of the limited angular resolution and sensitivity of the present observations, the derivation of the velocity fields and of the rotation curves is not straightforward. In particular the possible effects of beam smearing must be understood and corrected for (see also Bosma 1978; Begeman 1989; Rubin et al. 1989; Broeils 1992b; de Blok \& McGaugh 1997; Swaters 1999; Swaters et al. 2000; van den Bosch et al. 2000; van den Bosch \& Swaters 2001; McGaugh et al. 2001).

Due to the finite beam size, the H I emission will be smeared out, leading to apparently larger H I disks and to filling of holes in the H I distribution. Moreover, as a result of beam smearing the gradients in the velocity fields, as determined for example from Gaussian fits to the line profiles, may become shallower. Hence, rotation curves derived from these velocity fields have shallower gradients as well. The magnitude of the effect of beam smearing thus depends on the combination of the size of the beam, the distribution of the HI, the inclination angle of the galaxy and the intrinsic velocity gradients. Despite the effects of beam smearing, information on the true rotation curve is still contained in the data, and may be retrieved if an appropriate method to derive the rotation curve is used.

To take the effects of beam smearing into account, we have adopted a two-step procedure. First, we obtained the orientation parameters and an initial estimate of the rotation curve, if possible from a tilted ring fit, and using an interactive procedure if not. In the second step, this estimate of the rotation curve was refined iteratively by constructing model data cubes, using the input rotation curve and adjusting this rotation curve to match the model cubes to the observed ones.

Because for most galaxies the signal-to-noise ratios of the full resolution data were too low to derive reliable rotation curves, we have derived the rotation curves from the $30^{\prime \prime}$ data.

\subsubsection{Initial estimate of the rotation curves}

Before deriving the initial estimate of the rotation curve for a galaxy, we determined the position of its center. For most of the galaxies it was not possible to derive the center from the velocity field because of the often close to linear inner rise of the rotation curves. Therefore, we have used the optical centers from Paper II to define the centers, except when noted otherwise in Table 1.

Next, the systemic velocity was determined from a tiltedring fit to the Gaussian-fit velocity field, derived from the $30^{\prime \prime}$ velocity field (see Paper I), with the center fixed, by taking the average over all rings, except the outer rings which usually have large uncertainties. While determining the systemic velocity, the position angle was kept fixed at the initial estimate derived from 
Table 1. Galaxy centers.

\begin{tabular}{lrrl}
\hline \hline UGC & RA $(2000)$ & Dec. & Comments \\
\hline 731 & $1^{\mathrm{h}} 10^{\mathrm{m}} 43.0^{\mathrm{s}}$ & $49^{\circ} 36^{\prime} 7^{\prime \prime}$ & Kinematic center \\
2455 & $2^{\mathrm{h}} 59^{\mathrm{m}} 42.6^{\mathrm{s}}$ & $25^{\circ} 14^{\prime} 34^{\prime \prime}$ & Center of H H distribution \\
4173 & $8^{\mathrm{h}} 7^{\mathrm{m}} 8.7^{\mathrm{s}}$ & $80^{\circ} 7^{\prime} 38^{\prime \prime}$ & New optical center \\
4325 & $8^{\mathrm{h}} 19^{\mathrm{m}} 20.3^{\mathrm{s}}$ & $50^{\circ} 0^{\prime} 36^{\prime \prime}$ & Kinematic center \\
5414 & $10^{\mathrm{h}} 3^{\mathrm{m}} 57.2^{\mathrm{s}}$ & $40^{\circ} 45^{\prime} 22^{\prime \prime}$ & New optical center \\
5721 & $10^{\mathrm{h}} 32^{\mathrm{m}} 16.6^{\mathrm{s}}$ & $27^{\circ} 40^{\prime} 8^{\prime \prime}$ & Kinematic center \\
8188 & $13^{\mathrm{h}} 5^{\mathrm{m}} 51.4^{\mathrm{s}}$ & $37^{\circ} 35^{\prime} 56^{\prime \prime}$ & Center of H I distribution \\
8490 & $13^{\mathrm{h}} 29^{\mathrm{m}} 36.4^{\mathrm{s}}$ & $58^{\circ} 25^{\prime} 10^{\prime \prime}$ & Kinematic center \\
8837 & $13^{\mathrm{h}} 54^{\mathrm{m}} 43.2^{\mathrm{s}}$ & $53^{\circ} 53^{\prime} 42^{\prime \prime}$ & Center of H I distribution \\
\hline
\end{tabular}

the velocity field, and the inclination was kept fixed at the value derived from the ellipticity of the optical isophotes (see Paper II). We then made a new tilted ring fit with center and systemic velocity fixed to obtain the initial estimates for the rotation curve and the run of inclination and position angle with radius. If there were no appreciable variations of the inclination or position angle with radius, a fixed value was used (the average over all rings, excluding the rings with large uncertainties). If it was not possible to estimate the inclination from the velocity field, the inclination was determined from the ellipticity of the optical isophotes (see Paper II) or H I contours (see Paper I), assuming an intrinsic disk thickness of 0.2 .

Because many of the galaxies in our sample are small and affected by beam smearing, we found that in many cases it was difficult to obtain a good initial estimate of the rotation curve and orientation parameters from the tilted ring fits, especially in the central parts of the galaxies (see also de Blok et al. 1996). In those cases, we interactively fit the rotation curve and the orientation parameters to a set of six position-velocity diagrams ${ }^{1}$ as illustrated in Fig. 1 to improve the initial estimates (one along the major axis, two at a $30^{\circ}$ angle away from the major axis as measured in the plane of the galaxy, two $60^{\circ}$ degrees from the major axis, and one along the minor axis of the galaxy). To assure ourselves we are able to accurately retrieve the rotation curves and orientation parameters, we created several model galaxies with different and radially changing rotation velocities and orientation parameters. Except for pure solid-body rotators, we were able to retrieve the input parameters to within the uncertainties expected based on the noise added to the models.

In this interactive fitting, the rotation velocity, inclination and position angle at each radius were adjusted until the projected point was deemed to best match the six position-velocity diagrams simultaneously. Most weight was given to the major axis, and least to the minor axis. For determining the run of inclination and position angle with radius, equal weight was given to all position-velocity diagrams. Inclinations of around $30^{\circ}$ and lower are uncertain. We applied this method to the Hanning smoothed, $30^{\prime \prime}$ resolution data.

The inclinations and position angles as derived from the tilted rings fits or the interactive fits are listed in Table 2. For warped galaxies the orientation of the inner, unwarped part of the disks are given. For galaxies with clear differences between the approaching and receding sides, rotation curve were determined from both sides independently, using the same orientation parameters. The rotation curves have been sampled every

\footnotetext{
1 This interactive fitting was done with the task INSPECTOR, implemented in the Groningen Image Processing System (GIPSY, http:// wWw. astro.rug.nl/ gipsy)
}

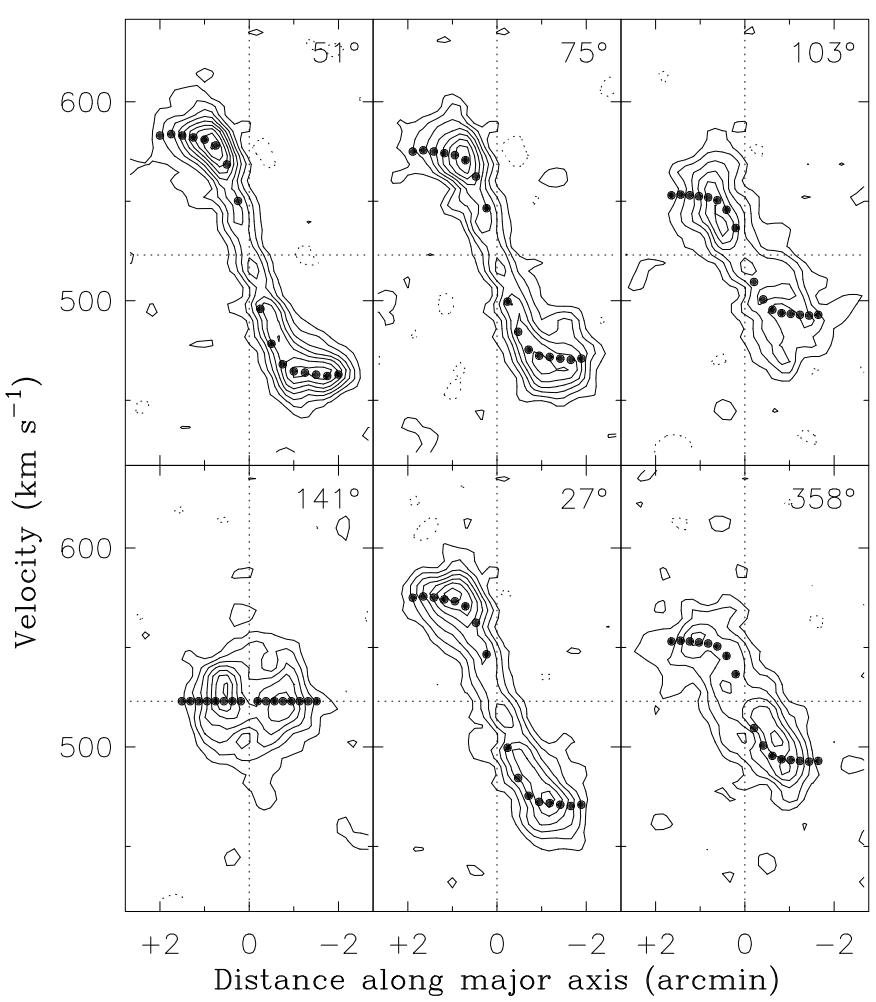

Fig. 1. Example of an interactively determined rotation curve (solid points) fit to six position-velocity diagrams (contours), which were taken along the position angels indicated in the top right of each panel. The galaxy shown here is UGC 4325 . To determine the rotation curve the position angle, the inclination and the rotation velocity were changed until the projected points on the position-velocity diagrams were found to best match the data.

15 arcsec, and the individual points in the rotation curves are therefore not independent.

For seven galaxies (UGC 4274, UGC 6817, UGC 7199, UGC 7408, UGC 8201, UGC 8331 and UGC 9128) we have not derived a rotation curve, because of a combination of small $\mathrm{H} \mathrm{I}$ extent $\left(R_{\mathrm{HI}} \lesssim 1^{\prime}\right)$, clumpy H I distribution and lack of ordered rotation.

\subsubsection{Final rotation curves}

A potential shortcoming of our method to derive the initial estimates of the rotation curves is that it is subjective. To obtain a more objective rotation curve and to investigate the effects of beam smearing further, we refined the initial estimate of the rotation curves by detailed modeling of the observations.

First, for each galaxy, an axisymmetric velocity field was constructed, based on the rotation curve determined as described above. Next, a three-dimensional model datacube was constructed from this velocity field and the observed $\mathrm{H}$ I distribution at the full resolution, assuming a constant Gaussian velocity dispersion of $8 \mathrm{~km} \mathrm{~s}^{-1}$. The model was subsequently convolved to a resolution of $30^{\prime \prime}$. The datacube obtained in this way was compared to the observations by calculating the $\chi^{2}$ value of the difference between model and observations over a small sub-cube that contained all H I emission, and also by visually comparing six model position-velocity diagrams (like those shown in Fig. 1) with those of the observations. Although we used the $\chi^{2}$ values to find better matching models, we have not used an algorithm to minimize $\chi^{2}$ because of the prohibitively large amounts of 


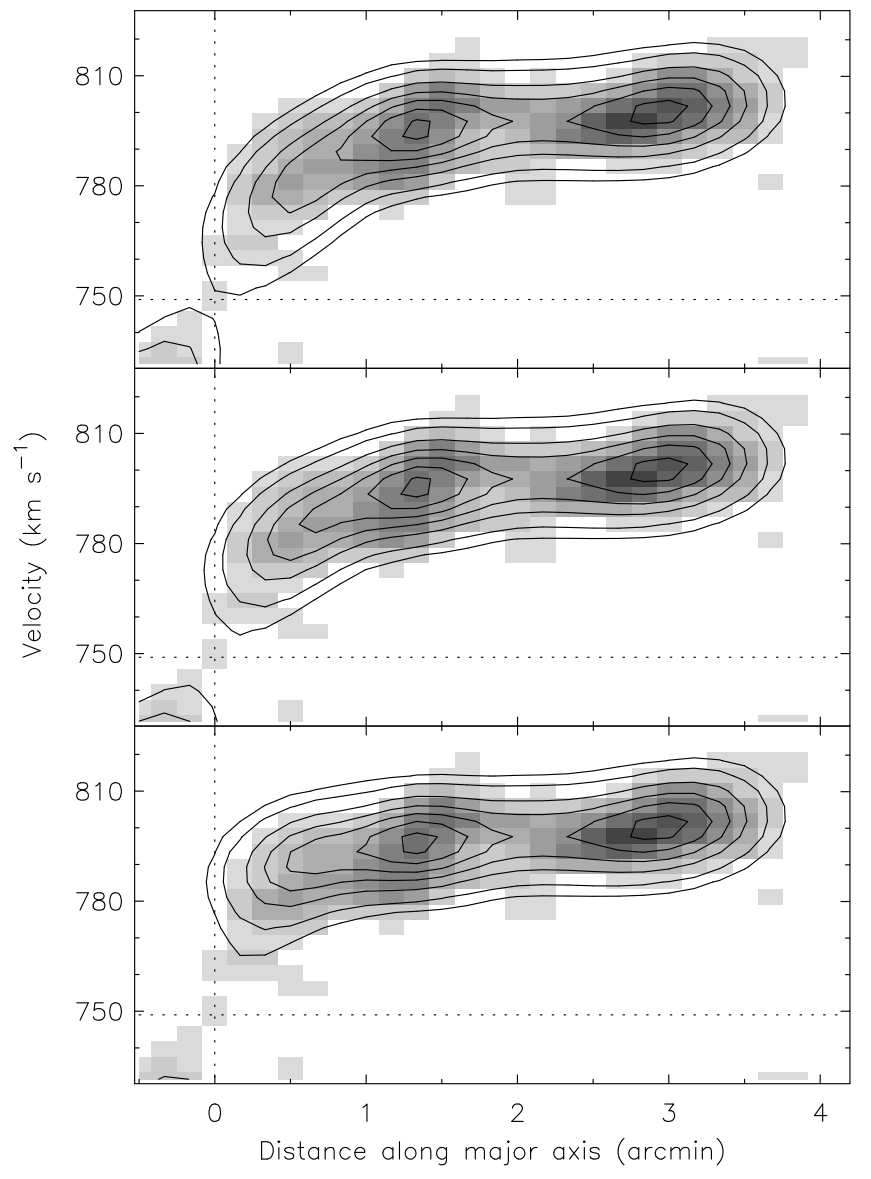

Fig. 2. Comparison between the data for the receding side of UGC 12732, shown in grayscale, and models of the observations (contours), based on an input rotation curve and the observed $\mathrm{H}$ I distribution at the full resolution. The input rotation curve is adjusted until the best match to the data is obtained (shown in the middle panel). The top panel shows the model based on the rotation curve derived by visual inspection, which underestimates the rotation velocities in the inner parts. The bottom panel shows a model based on a rotation curve that overestimates the rotation velocities between the center and $1^{\prime}$.

computing time needed. If the match between model and observations was not satisfactory, as determined from the visual inspection, the input rotation curve was adjusted interactively and the procedure was repeated until the $\chi^{2}$ had reached an approximate minimum. For most galaxies only the rotation velocities in the inner $60^{\prime \prime}$ needed to be adjusted. Position angles and inclinations have not been adjusted using this method. For galaxies with differences between the approaching and receding sides, the modeling was done for both sides independently.

To verify that our method works, we constructed model galaxies with known rotation curves, orientation parameters, and HI distributions, and derived the rotation curves with the method described here. As long as there was detectable HI emission in the center and the model galaxies were not close to edge-on, we were able to reproduce the rotation velocities accurate to a few $\mathrm{km} \mathrm{s}^{-1}$ (depending on the noise). Independent verification that the method works well is given in Sect. 5, where we compare our $\mathrm{H}$ I rotation curves with $\mathrm{H} \alpha$ rotation curves available in the literature.

An example of the modeling procedure is given in Fig. 2. The data for the receding side of UGC 12732 are shown in grayscale, and different models are overlayed in contours. The top panel shows the model based on the rotation curve determined interactively. Close inspection reveals that between the center and 1 arcmin the model fails to reproduce the observations. The model systematically underestimates the velocities in the central regions. In the middle panel, we show the model that we considered the best match, as found from the iterative modeling procedure described above. In the bottom panel, a model is shown based on a rotation curve for which the rotation velocities in the inner parts have been overestimated on purpose. This is also reflected in the model, which systematically overestimates the velocities between the center and $1^{\prime}$. Note that the comparison of the model and the observation was not done based on one position-velocity diagram, like the one shown in Fig. 2, but using the entire data cube for calculating $\chi^{2}$, and using six position-velocity diagrams.

The final rotation curves are shown in Appendix A. In the cases where the rotation curves have been derived for both sides independently, the average of the two is shown as well. The determination of the uncertainties in the derived rotation velocities is described below. In Appendix A the rotation curves are also shown overlayed on the position-velocity diagrams along the major axes. Where applicable, the rotation curves for the approaching and receding sides are overlayed rather than the average rotation curve.

\subsection{Rotation curves for edge-on galaxies}

In principle, it would have been possible to derive the rotation curves for the edge-on galaxies in our sample using a procedure similar to the modeling of NGC 1560 (Broeils 1992b) or NGC 891 (Swaters et al. 1997). However, such a procedure is extremely time-consuming, and therefore we did not use it. Instead, we chose to follow the approach used by Sancisi \& Allen (1979) to derive the rotation curve of NGC 891. At each position along the major axis the rotation velocity was determined from the edge of the line profile, correcting for the effects of instrumental broadening and random motions. The random motions of the gas were assumed to be $8 \mathrm{~km} \mathrm{~s}^{-1}$. In this method, it is assumed that there is gas everywhere along the line of nodes. Consequently, if a galaxy has a ring-like distribution of $\mathrm{H}$ I or a central depression in the H I distribution, an incorrect rotation curve may be derived $^{2}$.

The rotation curves for edge-on galaxies derived in this way for the approaching and receding sides separately are shown overlayed on the position-velocity diagrams along the major axis in Appendix A. The average rotation curves are shown in Appendix A as well.

\subsection{Uncertainties of the rotation velocities}

The method outlined above to derive the rotation curves does not provide an estimate of the uncertainties of the rotation velocities. Therefore, we have estimated the uncertainties resulting from the two main sources of error. The first is the accuracy with which the position of a point on the position-velocity diagrams can be measured. This measurement error depends on the width

\footnotetext{
2 After we derived the rotation curves for the galaxies in our sample, alternative methods to derive the rotation curves for edge-on galaxies have been published that are more objective (e.g., Kregel \& van der Kruit 2004; García-Ruiz et al. 2002). Because of the intrinsic uncertainties in the derivation of the rotation curves of edge-on galaxies, and given that we do not use the rotation curve for the edge-on galaxies in our analysis, we have not rederived the rotation curves.
} 
and the signal-to-noise ratio of the profile. Based on trial and error for a range of profiles, we have found that the $1 \sigma$ error with which the radial velocity can be determined from a profile is about $2 \mathrm{~km} \mathrm{~s}^{-1}$. The $1 \sigma$ errors for the points on the rotation curve is therefore $2 / \sin i \mathrm{~km} \mathrm{~s}^{-1}$.

The second source of error stems from non-circular motions and the asymmetry of the galaxy, which can be estimated by the difference between the approaching and the receding sides. We made the ad hoc assumption that the difference in rotation velocity between the mean rotation curve and the rotation curve measured on either the approaching or receding side represents a $2 \sigma$ difference. We have added the asymmetry error and the measurement error quadratically to obtain an estimate of the $1 \sigma$ errors in the rotation velocities.

For the edge-on galaxies we have used the same procedure to estimate the errors. This estimate does not include possible systematic effects as a result of lack of gas on the line of nodes. As a result, some of the rotation curves for the edge-on galaxies may be more uncertain than indicated by the errors. This systematic uncertainty was taken into account in determining the rotation curve quality, as described below.

\subsection{Rotation curve quality}

The quality of the derived rotation curves differs from galaxy to galaxy. Some galaxies are well resolved, others are only a few beams across. Some galaxies have strong H I emission, others are weak in H I. Some galaxies have distorted kinematics, others show ordered rotation. Hence, for some galaxies we could derive reliable rotation curves, whereas for others only lower quality rotation curves could be derived. We have tried to quantify this by tentatively dividing the derived rotation curves into four different categories of quality $q$. The quality depends on the signalto-noise ratio, on the presence of non-circular motions and on deviations from axial symmetry.

The quality estimates (tabulated in Table 2) range in value from 1 to 4 , where 1 indicates a rotation curve deemed to be reliable, 2 indicates an uncertain rotation curve, 3 indicates a highly uncertain rotation curve, and 4 means that no rotation curve could be derived, either because the HI distribution or kinematics were too irregular, or because there was no significant rotation.

\subsection{Asymmetric drift}

The stability of a galaxy against gravitational collapse is provided by rotation and pressure gradients. To derive the true circular velocity that reflects the underlying mass distribution, the observed rotation curve should in principle be corrected for the effects of pressure. The difference between the observed and the true circular velocity can be derived from the Jeans equations (Binney \& Tremaine 1987, Eqs. (4)-(33)) and is given by:

$v_{\varphi}^{2}-v_{\mathrm{c}}^{2}=\frac{R}{\rho} \frac{\partial P}{\partial R}$,

where $v_{\varphi}$ is the observed rotation velocity, $v_{\mathrm{c}}$ the true circular velocity, $R$ the radius, $\rho$ the density at that radius, and $P$ the pressure. The pressure can also be written as $\rho \sigma^{2}$, where $\sigma$ is the one-dimensional random velocity of the gas. Inserting this in Eq. (1), one obtains:

$v_{\mathrm{c}}^{2}=v_{\varphi}^{2}-\sigma^{2}\left(\frac{\partial \ln \rho}{\partial \ln R}+\frac{\partial \ln \sigma^{2}}{\partial \ln R}\right)$.
We found that the velocity dispersions for the late-type dwarf galaxies in this sample were almost constant with radius, hence the second term within parentheses in Eq. (2) can be ignored. If we assume that the scale height is approximately constant with radius as well (as is suggested by measurements of the thickness of the Hi layer in the edge-on dwarf galaxy NGC 5023, Bottema et al. 1986), and if we assume that the H I is the dominant gaseous component, the volume density is proportional to the measured $\mathrm{H}$ I column density.

With these assumptions, the correction for asymmetric drift is smaller than $3 \mathrm{~km} \mathrm{~s}^{-1}$ at all radii, for all but three galaxies. For most galaxies the maximum correction occurs at the last measured point, where the amplitude of the rotation curve is highest. In the inner parts the correction is negligible, i.e., smaller than $1 \mathrm{~km} \mathrm{~s}^{-1}$, for $90 \%$ of the galaxies, and smaller than $2 \mathrm{~km} \mathrm{~s}^{-1}$ for all except three galaxies. These are the same galaxies for which the maximum correction is larger than $3 \mathrm{~km} \mathrm{~s}^{-1}$ : UGC 7278 , UGC 7577 and UGC 8331.

Because the corrections for asymmetric drift as calculated above are small for the vast majority of the galaxies in our sample, and because of the uncertainties in the corrections due to ignoring the contribution of other gas components, we have not corrected the rotation curves for asymmetric drift, and we assumed that our derived rotation curves corrected for inclination represent the circular velocity.

\subsection{Parametrized rotation curve shapes}

The radial scale of the rotation curves derived above is given in arcseconds. In order to facilitate the comparison of the rotation curves between galaxies and the comparison between the rotation curves and the light profiles, the rotation curves have been resampled in units of disk scale lengths. Table 2 lists the rotation velocities at one, two, three, and four disk scale lengths, as $v_{1}$ to $v_{4}$. In addition, it lists the velocity at the last measured point of the rotation curve $v_{\text {last }}$ and the corresponding radius $r_{\text {last }}$.

We have also calculated the logarithmic slope of the rotation curves. The logarithmic slope is defined as a straight line fit to the rotation curve in logarithmic coordinates between two radii:

$S_{(\mathrm{a}, \mathrm{b})}=\frac{\Delta \log v}{\Delta \log R}=\frac{\log \left(v_{b} / v_{a}\right)}{\log \left(R_{b} / R_{a}\right)}$.

For a flat rotation curve, $S_{(a, b)}$ equals zero, and for a solid body rotation curve $S_{(a, b)}$ equals unity. In Table 2, we list $S_{(1,2)}^{h}, S_{(2,3)}^{h}$ and $S_{(2, \mathrm{~L})}^{h}$, where the superscript $h$ refers to the fact that the logarithmic slopes have been calculated from the rotation curves expressed in units of disk scale length. The subscript numbers between parentheses refer to the number of scale lengths, and the " $L$ " refers to the radius of the last measured point.

\section{Notes on individual galaxy rotation curves}

Because of the relatively large size of the sample, we restrict ourselves here to a discussion of properties relevant to the derivation of the rotation curves.

UGC 2034 has a small kinematic warp. The position angle changes by about $35^{\circ}$. Because the galaxy is almost face-on, the variation of inclination angle with radius is difficult to determine. UGC 2053 has its kinematic major axis and its morphological major axis almost at right angles. Its kinematics are reminiscent of that expected for a bar potential. The bright optical image appears to have a bar-like shape, but no faint disk is detected, neither in the optical nor in the HI. 
Table 2. Optical and rotation curve properties.

\begin{tabular}{|c|c|c|c|c|c|c|c|c|c|c|c|c|c|c|c|c|c|c|}
\hline UGC & $\begin{array}{r}D_{a} \\
\mathrm{Mpc} \\
(2)\end{array}$ & $\begin{array}{r}M_{R} \\
\mathrm{mag} \\
(3)\end{array}$ & Type & $\begin{array}{r}h \\
\mathrm{kpc} \\
(5)\end{array}$ & $\begin{array}{r}\mu_{0}^{R} \\
\mathrm{mag}^{\prime \prime-2} \\
\text { (6) }\end{array}$ & (7) & (8) & $\begin{array}{r}\text { PA } \\
\circ \\
(9)\end{array}$ & $\begin{array}{c}v_{\text {sys }} \\
- \\
(10)\end{array}$ & $\begin{array}{c}v_{1} \\
- \\
(11)\end{array}$ & $\begin{array}{c}v_{2} \\
-\quad \mathrm{km} \\
(12)\end{array}$ & $\begin{array}{l}\mathrm{s}^{-1}- \\
(13)\end{array}$ & $\begin{array}{l}v_{4} \\
-\quad- \\
(14)\end{array}$ & $\begin{array}{c}v_{\text {last }} \\
- \\
(15)\end{array}$ & $\begin{array}{l}r_{\text {last }} \\
\mathrm{kpc} \\
(16)\end{array}$ & $S_{(1,2)}$ & $S_{(2,3)}$ & $S_{(2, \mathrm{~L})}$ \\
\hline 731 & 8.0 & -16.6 & .IB.9\$. & 1.65 & 23.0 & 1 & 57 & 257 & 638 & 50 & 63 & 73 & 74 & 74 & 6.98 & 0.35 & 0.34 & 0.20 \\
\hline 1281 & 5.5 & -16.2 & .S..8.. & 1.66 & 22.7 & 2 & 90 & 220 & 157 & 32 & 53 & 57 & - & 57 & 5.20 & 0.73 & 0.15 & 0.14 \\
\hline 2023 & 10.1 & -17.2 & .I..9*. & 1.22 & 21.8 & 2 & 19 & 315 & 603 & 24 & 41 & 59 & - & 59 & 3.67 & 0.77 & 0.90 & 0.90 \\
\hline 2034 & 10.1 & -17.4 & .I..9.. & 1.29 & 21.6 & 1 & 19 & 342 & 578 & 29 & 37 & 40 & 45 & 47 & 5.88 & 0.39 & 0.18 & 0.29 \\
\hline 2053 & 11.8 & -16.0 & .I..9.. & 1.09 & 22.5 & 3 & 40 & 323 & 1025 & 62 & 85 & 97 & - & 99 & 3.43 & 0.45 & 0.32 & 0.33 \\
\hline 2455 & 7.8 & -18.5 & .IBS9.. & 1.06 & 19.8 & 2 & 51 & 263 & 371 & 23 & 32 & 42 & 57 & 61 & 4.54 & 0.45 & 0.68 & 0.87 \\
\hline 3137 & 18.4 & -18.7 &.$S ? \ldots$ & 1.95 & 24.2 & 2 & 90 & 74 & 993 & 31 & 66 & 94 & 103 & 100 & 30.8 & 1.08 & 0.88 & 0.20 \\
\hline 3371 & 12.8 & -17.7 &. I.. $9 *$. & 3.09 & 23.3 & 1 & 49 & 133 & 818 & 51 & 75 & 81 & - & 86 & 10.2 & 0.55 & 0.18 & 0.26 \\
\hline 3698 & 8.5 & -15.4 & .I..9*. & 0.41 & 21.2 & 3 & 40 & 334 & 420 & 51 & 75 & - & - & 27 & 1.24 & 0.55 & - & - \\
\hline 3711 & 8.6 & -17.8 & .IB.9.. & 0.96 & 20.9 & 2 & 60 & 281 & 433 & 79 & 92 & 93 & - & 95 & 3.75 & 0.21 & 0.03 & 0.05 \\
\hline 3817 & 8.7 & -15.1 & .I..9*. & 0.71 & 22.5 & 1 & 30 & 0 & 436 & 16 & 29 & 38 & - & 45 & 2.53 & 0.82 & 0.70 & 0.78 \\
\hline 3851 & 3.4 & -16.9 & .IBS9.. & 1.31 & 22.6 & 2 & 59 & 42 & 104 & 31 & 50 & 55 & 54 & 60 & 5.85 & 0.70 & 0.21 & 0.22 \\
\hline 3966 & 6.0 & -14.9 & .I..9.. & 0.58 & 22.2 & 2 & 41 & 270 & 364 & 38 & 46 & 47 & - & 50 & 2.18 & 0.28 & 0.07 & 0.12 \\
\hline 4173 & 16.8 & -17.8 & .I..9*. & 4.46 & 24.3 & 2 & 40 & 168 & 865 & 36 & 49 & - & - & 57 & 12.2 & 0.46 & - & 0.44 \\
\hline 4274 & 6.6 & -17.9 & .SBS9P. & 0.71 & 20.7 & 4 & 00 & 0 & 447 & - & - & - & - & - & - & - & - & - \\
\hline 4278 & 10.5 & -17.7 &.$S B S 7 *$ & 1.42 & 22.5 & 2 & 90 & 353 & 559 & 29 & 53 & 65 & 77 & 86 & 7.64 & 0.89 & 0.51 & 0.48 \\
\hline 4305 & 3.4 & -16.8 & .I..9.. & 1.04 & 21.7 & 2 & 40 & 172 & 156 & 24 & 37 & 33 & 33 & 33 & 5.44 & 0.62 & -0.26 & -0.11 \\
\hline 4325 & 10.1 & -18.1 & .SAS9\$. & 1.63 & 21.6 & 1 & 41 & 51 & 523 & 73 & 89 & 92 & - & 92 & 5.88 & 0.29 & 0.08 & 0.04 \\
\hline 4499 & 13.0 & -17.8 & .SX.8.. & 1.49 & 21.5 & 1 & 50 & 140 & 691 & 38 & 58 & 66 & 71 & 74 & 8.51 & 0.63 & 0.30 & 0.24 \\
\hline 4543 & 30.3 & -19.2 & .SA.8.. & 4.00 & 22.0 & 2 & 46 & 331 & 1956 & 59 & 61 & 64 & 66 & 67 & 17.6 & 0.03 & 0.12 & 0.13 \\
\hline 5272 & 6.1 & -15.1 &. .I..9.. & 0.60 & 22.4 & 2 & 59 & 97 & 525 & 19 & 33 & - & - & 45 & 1.78 & 0.81 & - & 0.83 \\
\hline 5414 & 10.0 & -17.6 & .IXS9.. & 1.49 & 21.8 & 1 & 55 & 220 & 607 & 35 & 52 & - & - & 61 & 4.36 & 0.57 & - & 0.42 \\
\hline 5721 & 6.7 & -16.6 &.$S X .7 ?$ & 0.45 & 20.2 & 2 & 61 & 279 & 542 & 39 & 54 & 68 & 78 & 79 & 7.31 & 0.46 & 0.59 & 0.18 \\
\hline 5829 & 9.0 & -17.3 & .I..9.. & 1.94 & 22.4 & 2 & 34 & 197 & 627 & 34 & 48 & 60 & - & 69 & 7.20 & 0.49 & 0.59 & 0.59 \\
\hline 5846 & 13.2 & -16.1 & .I..9.. & 1.18 & 22.9 & 2 & 30 & 305 & 1019 & 30 & 46 & 51 & - & 51 & 3.84 & 0.63 & 0.24 & 0.19 \\
\hline 5918 & 7.7 & -15.4 & .I..9*. & 1.27 & 24.2 & 2 & 46 & 239 & 337 & 30 & 38 & 42 & - & 45 & 4.48 & 0.38 & 0.24 & 0.26 \\
\hline 5986 & 8.7 & -18.6 & .SBS9. & 2.18 & 21.4 & 2 & 90 & 219 & 624 & 76 & 112 & 116 & 110 & 125 & 12.0 & 0.56 & 0.10 & 0.11 \\
\hline 6446 & 12.0 & -18.4 & .SA.7.. & 1.87 & 21.4 & 1 & 52 & 188 & 647 & 58 & 70 & 75 & 78 & 80 & 9.60 & 0.27 & 0.17 & 0.15 \\
\hline 6628 & 15.3 & -18.9 & .SA.9.. & 2.70 & 21.8 & 2 & 20 & 204 & 851 & 40 & 42 & - & - & 42 & 7.79 & 0.08 & - & 0.01 \\
\hline 6817 & 4.02 & -15.2 & .I..9.. & 0.97 & 23.1 & 4 & 60 & 140 & 243 & - & - & - & - & - & - & - & - & - \\
\hline 6956 & 15.7 & -17.2 & .SBS9.. & 2.37 & 23.4 & 3 & 30 & 118 & 917 & 34 & 49 & - & - & 58 & 5.71 & 0.52 & - & 0.82 \\
\hline 7047 & 3.5 & -15.2 & .IA.9.. & 0.48 & 21.6 & 2 & 46 & 34 & 208 & 16 & 26 & 36 & - & 38 & 1.53 & 0.70 & 0.86 & 0.85 \\
\hline 7125 & 19.5 & -18.3 & $. S . .9 .$. & 1.73 & 22.8 & 2 & 90 & 84 & 1078 & 30 & 46 & 51 & 56 & 70 & 21.3 & 0.61 & 0.23 & 0.23 \\
\hline 7151 & 3.5 & -15.7 & .SXS6\$ & 0.54 & 22.3 & 2 & 90 & 282 & 267 & 44 & 64 & 68 & 72 & 76 & 2.80 & 0.53 & 0.12 & 0.18 \\
\hline 7199 & 3.5 & -15.1 & .IA.9.. & 0.37 & 21.4 & 4 & 50 & 192 & 166 & - & - & - & - & - & - & - & - & - \\
\hline 7232 & 3.5 & -15.3 & .I..9P. & 0.33 & 20.2 & 2 & 59 & 0 & 230 & 19 & 32 & 43 & - & 44 & 1.02 & 0.71 & 0.76 & 0.76 \\
\hline 7261 & 9.1 & -17.7 & .SBS8.. & 1.69 & 21.9 & 2 & 30 & 262 & 856 & 66 & 74 & - & - & 76 & 4.63 & 0.16 & - & 0.11 \\
\hline
\end{tabular}

UGC 3711 has twisted isovelocity contours. Because this galaxy is poorly resolved, it is difficult to determine the cause; the twisted contours may indicate a kinematic warp, but may also be related to the bar-shaped morphology.

UGC 3851 has an irregular kinematic structure. On the north side, the isovelocity contours curve strongly, suggesting a nearly flat rotation curve. On the south side, the isovelocity contours are almost straight, and not symmetric with respect to the major axis, but instead run more or less north to south.

UGC 4173 has a faint optical disk surrounding a central bar-like structure. The kinematical major axis is aligned with the faint disk. The bar is not clearly reflected in the velocity field.
UGC 4274 is asymmetric and $\mathrm{H} \mathrm{I}$ is only detected in one clump on the receding side. No rotation curve has been derived.

UGC 4305 has previously been found to have a violent interstellar medium (e.g., Puche et al. 1992). The observations presented here also show signs of holes, shells, and small-scale kinematic irregularities, making an accurate determination of the rotation curve difficult.

UGC 5272 displays a misalignment between the optical and the kinematic major axes.

UGC 5721 has the most extended rotation curve of this sample when the radius is expressed in optical disk scale lengths. The last measured point of the rotation curve is at $16.3 h$. The inner 
Table 2. continued.

\begin{tabular}{|c|c|c|c|c|c|c|c|c|c|c|c|c|c|c|c|c|c|c|}
\hline UGC & $\begin{array}{r}D_{\mathrm{D}} \\
\mathrm{Mpc} \\
(2)\end{array}$ & $\begin{array}{r}M_{R} \\
\text { mag } \\
(3)\end{array}$ & type & $\begin{array}{r}h \\
\mathrm{kpc} \\
(5)\end{array}$ & $\begin{array}{r}\mu_{0}^{R} \\
\operatorname{mag}^{\prime \prime-2} \\
\text { (6) }\end{array}$ & (7) & (8) & $\begin{array}{r}\text { PA } \\
\circ \\
(9)\end{array}$ & $\begin{array}{c}v_{\text {sys }} \\
- \\
(10)\end{array}$ & $\begin{array}{c}v_{1} \\
- \\
(11)\end{array}$ & $\begin{array}{r}\mathrm{km} \\
(12)\end{array}$ & $\begin{array}{l}\mathrm{s}^{-1}{ }^{v_{3}} \\
\text { (13) }\end{array}$ & $\begin{array}{l}v_{4} \\
-\quad- \\
(14)\end{array}$ & $\begin{array}{c}v_{\text {last }} \\
- \\
(15)\end{array}$ & $\begin{array}{l}r_{\text {last }} \\
\mathrm{kpc} \\
(16)\end{array}$ & $S_{(1,2)}$ & $S_{(2,3)}$ & $S_{(2, \mathrm{~L})}$ \\
\hline 7278 & 3.5 & -18.3 & .IXS9.. & 0.93 & 20.2 & 2 & 30 & 74 & 292 & 47 & 68 & 75 & 79 & 81 & 5.80 & 0.51 & 0.26 & 0.15 \\
\hline 7323 & 8.1 & -18.9 & .SXS8.. & 2.20 & 21.2 & 1 & 47 & 38 & 518 & 49 & 78 & - & - & 86 & 5.89 & 0.66 & - & 0.33 \\
\hline 7399 & 8.4 & -17.1 & .SBS8.. & 0.79 & 20.7 & 1 & 55 & 320 & 535 & 55 & 79 & 89 & 92 & 109 & 11.0 & 0.54 & 0.27 & 0.16 \\
\hline 7408 & 8.4 & -16.6 & .IA.9.. & 0.99 & 21.9 & 4 & 45 & 275 & 462 & - & - & - & - & - & - & - & - & - \\
\hline 7490 & 8.5 & -17.4 & .SA.9.. & 1.19 & 21.3 & 3 & 20 & 113 & 464 & 57 & 71 & - & - & 79 & 3.09 & 0.32 & - & 0.42 \\
\hline 7524 & 3.5 & -18.1 & .SAS9 $*$. & 2.58 & 22.2 & 1 & 46 & 327 & 320 & 58 & 75 & 83 & - & 79 & 7.89 & 0.38 & 0.24 & 0.12 \\
\hline 7559 & 3.2 & -13.7 & .IB.9.. & 0.67 & 23.8 & 1 & 61 & 137 & 216 & 21 & 31 & 33 & - & 33 & 2.10 & 0.57 & 0.15 & 0.15 \\
\hline 7577 & 3.5 & -15.6 & .I..9.. & 0.84 & 22.5 & 1 & 63 & 128 & 196 & 8 & 13 & - & - & 18 & 2.29 & 0.78 & - & 0.92 \\
\hline 7603 & 6.8 & -16.9 & .SBS7? & 0.90 & 20.8 & 1 & 78 & 197 & 644 & 30 & 47 & 59 & 60 & 64 & 5.94 & 0.65 & 0.54 & 0.25 \\
\hline 7608 & 8.4 & -16.4 & .I..9.. & 1.24 & 22.6 & 1 & 25 & 257 & 535 & 32 & 51 & 62 & - & 69 & 4.89 & 0.67 & 0.48 & 0.46 \\
\hline 7690 & 7.9 & -17.0 &. I..9*. & 0.54 & 19.9 & 2 & 41 & 41 & 536 & 44 & 59 & 61 & 59 & 56 & 4.02 & 0.41 & 0.10 & -0.04 \\
\hline 7866 & 4.8 & -15.2 & .IXS9.. & 0.57 & 22.1 & 2 & 44 & 338 & 354 & 17 & 25 & 28 & 32 & 33 & 2.44 & 0.54 & 0.32 & 0.39 \\
\hline 7916 & 8.4 & -14.9 & .I..9.. & 1.81 & 24.4 & 2 & 74 & 0 & 603 & 21 & 33 & - & - & 36 & 4.28 & 0.62 & - & 0.57 \\
\hline 7971 & 8.4 & -17.1 & $. S . .9 *$ & 0.99 & 21.3 & 2 & 38 & 35 & 470 & 22 & 37 & 45 & - & 45 & 3.06 & 0.73 & 0.51 & 0.50 \\
\hline 8188 & 4.7 & -17.4 & .SAS9.. & 1.16 & 21.3 & 3 & 20 & 0 & 318 & 28 & - & - & - & 45 & 2.05 & - & - & - \\
\hline 8201 & 4.9 & -15.8 & .I..9.. & 0.80 & 21.9 & 4 & 63 & 90 & 30 & - & - & - & - & - & - & - & - & - \\
\hline 8286 & 4.8 & -17.2 & $. S . .6 *$ & 1.23 & 20.9 & 2 & 90 & 28 & 407 & 66 & 76 & 82 & 83 & 84 & 5.94 & 0.20 & 0.18 & 0.12 \\
\hline 8331 & 5.9 & -15.1 & .IA.9.. & 0.70 & 22.9 & 4 & 90 & 139 & 262 & 4 & 7 & 11 & 13 & 21 & 4.29 & 0.96 & 0.89 & 0.92 \\
\hline 8490 & 4.9 & -17.3 & .SAS9.. & 0.66 & 20.5 & 1 & 50 & 175 & 201 & 48 & 66 & 74 & 77 & 78 & 10.7 & 0.45 & 0.29 & 0.08 \\
\hline 8550 & 5.3 & -15.6 & .SBS7? & 0.67 & 22.0 & 2 & 90 & 166 & 358 & 39 & 49 & 51 & 53 & 58 & 4.24 & 0.33 & 0.10 & 0.14 \\
\hline 8683 & 12.6 & -16.7 & .I..9.. & 1.37 & 22.5 & 3 & 28 & 349 & 659 & 17 & 31 & - & - & 31 & 2.75 & 0.87 & - & 0.84 \\
\hline 8837 & 5.1 & -15.7 & .IBS9. & 1.63 & 23.2 & 2 & 80 & 22 & 135 & 31 & 52 & - & - & 54 & 3.71 & 0.77 & - & 0.22 \\
\hline 9128 & 4.4 & -14.3 & .I..9.. & 0.36 & 21.9 & 4 & 40 & 135 & 155 & - & - & - & - & - & - & - & - & - \\
\hline 9211 & 12.6 & -16.2 & I...9*. & 1.32 & 22.6 & 1 & 44 & 287 & 686 & 35 & 53 & 63 & 66 & 65 & 8.25 & 0.59 & 0.41 & 0.17 \\
\hline 9992 & 10.4 & -15.9 & .I..9.. & 0.75 & 22.2 & 2 & 30 & 35 & 428 & 28 & 31 & 33 & 33 & 34 & 3.78 & 0.15 & 0.13 & 0.10 \\
\hline 10310 & 15.6 & -17.9 & .SBS9.. & 1.66 & 22.0 & 1 & 34 & 199 & 718 & 44 & 65 & 70 & 72 & 74 & 9.08 & 0.57 & 0.17 & 0.13 \\
\hline 11557 & 23.8 & -19.7 & .SXS8.. & 3.10 & 21.0 & 2 & 30 & 274 & 1386 & 53 & 76 & 83 & - & 85 & 10.4 & 0.52 & 0.20 & 0.20 \\
\hline 11707 & 15.9 & -18.6 & .SA.8.. & 4.30 & 23.1 & 1 & 68 & 57 & 904 & 68 & 89 & 94 & - & 100 & 15.0 & 0.39 & 0.14 & 0.20 \\
\hline 11861 & 25.1 & -20.8 & .SX.8.. & 6.06 & 21.4 & 1 & 50 & 219 & 1481 & 111 & 147 & - & - & 153 & 16.4 & 0.41 & - & 0.12 \\
\hline 12060 & 15.7 & -17.9 & .IB.9.. & 1.76 & 21.6 & 1 & 40 & 183 & 883 & 61 & 72 & 74 & 75 & 74 & 10.3 & 0.24 & 0.07 & 0.03 \\
\hline 12632 & 6.9 & -17.1 & $. S . .9 *$. & 2.57 & 23.5 & 1 & 46 & 36 & 421 & 58 & 69 & 74 & - & 76 & 8.53 & 0.27 & 0.16 & 0.17 \\
\hline 12732 & 13.2 & -18.0 & $. S . .9 *$. & 2.21 & 22.4 & 1 & 39 & 14 & 749 & 53 & 68 & 76 & 81 & 98 & 15.4 & 0.35 & 0.27 & 0.29 \\
\hline
\end{tabular}

(1) UGC number; (2) the adopted distance, from Paper II; (3) the absolute $R$-band as determined in Paper II; (4) the morphological type according to the RC3 catalog (de Vaucouleurs et al. 1991), using the same coding; (5) and (6) the disk scale length and the $R$-band central disk surface brightness as determined in Paper II; (7) the rotation curve quality $q$ as defined in Sect. 3; (8) and (9) the adopted inclination and position angles; (10) the systemic velocity derived from the velocity field; (11) to (14) the rotation velocities at one, two, three and four disk scale lengths; (15) and (16) the rotation velocity at the last measured point and the radius of the last measured point; (17), (18) and (19) the logarithmic shape of the rotation curve between one and two disk scale lengths, between two and three disk scale lengths, and between two disk scale lengths and the last measured point.

points of this rotation curve are uncertain, because of insufficient angular resolution.

UGC 5986 is warped and has a strong asymmetry between the approaching and the receding side, both in kinematics and in density. A possible cause is an interaction with a small companion to the south west.

UGC 6817 has a clumpy and irregular H I distribution and kinematics. No rotation curve could be derived.

UGC 7199 has little H I and is poorly resolved by the $30^{\prime \prime}$ beam. No rotation curve could be derived.

UGC 7261 is strongly barred. The S-shape distortion in the kinematics that is associated with the bar is clearly visible in the velocity field.
UGC 7278 has a strong kinematic warp, with the inclination changing from about $30^{\circ}$ in the central parts to about $10^{\circ}$ in the outer parts, and the position angle changing from $74^{\circ}$ to $90^{\circ}$. Because of the low inclination, the actual values of the inclination, and hence the amplitude of the rotation curve, are uncertain. The H I in the central parts has a high velocity dispersion and seems to counterrotate with respect to the outer parts.

UGC 7399 has an extended H I rotation curve, yet the inner rise of the rotation curve is poorly resolved because of the small size of the optical galaxy.

UGC 7408 has little H I. No rotation curve could be derived.

UGC 7577 has the lowest rotation amplitude of the galaxies in this sample, yet its velocity field is still regular in appearance. 
UGC 7524 is a prototype of a kinematically lopsided galaxy. The rotation curve on the receding side continues to rise, whereas on the approaching side the rotation curve quickly reaches a flat part (see also Swaters et al. 1999).

UGC 7603 has a high inclination of $78^{\circ}$. As a result, the inner parts are effectively seen edge-on by the $30^{\prime \prime}$ beam, making the inner rotation curve more uncertain.

UGC 7690 appears to have a declining rotation curve. However, this galaxy also has a modest kinematic warp, with an inclination changing from about $40^{\circ}$ in the central parts to $30^{\circ}$ in the outer parts. Because the inclination as a function of radius is uncertain, so is the shape of the rotation curve.

UGC 8201 has most of its Hi located in two clumps that are roughly located on the major axis, but that show clear difference in velocity. No rotation curve could be derived.

UGC 8331 has $\mathrm{H}$ I in one clump in the center and two clumps at larger radii, making it impossible to measure the rotation curve. UGC 8490 is strongly warped. The position angle changes from $160^{\circ}$ to $225^{\circ}$, the inclination changes from $50^{\circ}$ to $30^{\circ}$, and then rises again to about $50^{\circ}$. This system was studied in detail by Sicotte et al. (1996) and Sicotte \& Carignan (1997). The outer half of the rotation curve may be more uncertain than indicated by the error bars, due to uncertainties in the orientation angles. UGC 9128 is hardly resolved by the $30^{\prime \prime}$ beam, and hence no rotation curve could be determined.

\section{Comparison to $\mathrm{H} \alpha$ rotation curves}

In Fig. 3 we compare the H I rotation curves presented here to high-resolution rotation curves based on long-slit $\mathrm{H} \alpha$ spectroscopy from the studies of $\mathrm{dBB}$ and $\mathrm{SMvdBB}$. There is a significant overlap between the samples of those two studies and the sample presented here because they are both based on the sample of Swaters (1999).

$\mathrm{dBB}$ have derived their rotation curves by combining their $\mathrm{H} \alpha$ data with the S99 H I data, and then fitting a spline to the combined data to obtain a smooth curve. The dBB data plotted in Fig. 3 are the points from their spline-interpolated rotation curves over the range in radii where they have $\mathrm{H} \alpha$ data.

The comparison between the $\mathrm{HI}$ and $\mathrm{H} \alpha$ rotation curves shows that they generally agree fairly well, although there are significant differences in individual galaxies. Below we discuss the comparison of the $\mathrm{H}$ I and $\mathrm{H} \alpha$ rotation curves in detail.

UGC 731. Observed in $\mathrm{H} \alpha$ both by SMvdBB and $\mathrm{dBB}$. The agreement between the $\mathrm{HI}$ data and the SMVdBB data is good, whereas the $\mathrm{H} \alpha$ rotation curve by de $\mathrm{dBB}$ rises more steeply than the HI rotation curve. The difference between these two $\mathrm{H} \alpha$ curves can be explained by the lopsided kinematics of this galaxy (see Swaters et al. 1999). Its approaching side has a more steeply rising rotation curve than the receding side. The $\mathrm{H} \alpha$ data in SMvdBB samples both sides, whereas in the $\mathrm{dBB}$ data, which is less deep, $\mathrm{H} \alpha$ emission is biased to the approaching side.

UGC 1281. The $\mathrm{HI}$ and $\mathrm{H} \alpha$ rotation curves for this edge-on galaxy are in excellent agreement.

UGC 3137. The $\mathrm{H} \alpha$ rotation curve rises more steeply than the H I one. This galaxy is edge-on, and has a rotation curve that rises steeply with respect to the size of the H I beam. This, in combination with its inclination, makes the $\mathrm{H}$ I rotation curve hard to determine.

UGC 3371. The $\mathrm{H}$ I rotation curve rises more steeply than the $\mathrm{H} \alpha$ rotation curve. The reason for this difference is unclear. Swaters et al. (2003b) obtained an $\mathrm{H} \alpha$ rotation curve derived from integral field spectroscopy and find a good match between their $\mathrm{H} \alpha$ rotation curve and our H I rotation curve.
UGC 3851. As noted above, this galaxy has an irregular kinematic structure (see also Hunter et al. 2002). The difference between the $\mathrm{HI}$ and $\mathrm{H} \alpha$ curves could be caused by the fact that the H I rotation curve is derived from the entire velocity field, whereas the $\mathrm{H} \alpha$ rotation curve is derived from a single, $1^{\prime \prime}$ wide slice along the major axis.

$U G C$ 4173. The $\mathrm{HI}$ and $\mathrm{H} \alpha$ rotation curves are in excellent agreement. However, the $\mathrm{H} \alpha$ data by $\mathrm{dBB}$ is of low quality, and their interpolated rotation curve is dominated by the $\mathrm{H}$ I data.

UGC 4278. The H I rotation curve of this edge-on galaxy rises more steeply than the $\mathrm{H} \alpha$ one.

UGC 4325. This galaxy has been observed by both SMvdBB and $\mathrm{dBB}$. The SMvdBB rotation curve is in good agreement with the $\mathrm{H}$ I curve, whereas the $\mathrm{dBB}$ curve rises more slowly and reaches a higher amplitude. The latter is difficult to understand, as the amplitude of the rotation curve can be determined accurately from the two-dimensional velocity field. A possible explanation is that $\mathrm{dBB}$ picked up a localized disturbance in the kinematics. UGC 4499. The $\mathrm{H} \alpha$ rotation curve has considerable scatter, but there is good general agreement between the $\mathrm{H} \mathrm{I}$ and $\mathrm{H} \alpha$ curves. UGC 5272. The $\mathrm{H} \mathrm{I}$ and $\mathrm{H} \alpha$ are in excellent agreement.

UGC 5721. This galaxy has been observed by both SMvdBB and $\mathrm{dBB}$. Both $\mathrm{H} \alpha$ curves agree well with each other, and both show a steep rise in the inner $10^{\prime \prime}$ that is not seen in the H I data. UGC 7524. This is the best resolved galaxy in our sample, yet the $\mathrm{H}$ I and $\mathrm{H} \alpha$ rotation curves show significant differences. The velocity field of this galaxy shows a great deal of structure, including kinematic lopsidedness (see Swaters et al. 1999). The structure seen in the $\mathrm{dBB}$ curve is also seen along the major axis of the H I data.

UGC 7603. The $\mathrm{H} \mathrm{I}$ and $\mathrm{H} \alpha$ data are in good agreement, although the outer points on the $\mathrm{H} \alpha$ curve fall below the $\mathrm{H}$ I curve. As this galaxy has a fairly high inclination $\left(76^{\circ}\right)$, and a steeply rising rotation curve that is not well resolved by the $\mathrm{H} \mathrm{I}$ beam, it is likely that the difference is due to residual affects of beam smearing. UGC 8286. The $\mathrm{HI}$ and $\mathrm{H} \alpha$ rotation curves for this edge-on galaxy agree except in the region where the rotation curve turns over.

UGC 8490. The $\mathrm{H} \alpha$ rotation curve falls well below the $\mathrm{H}$ I rotation curve near a radius of about $15^{\prime \prime}$. As this corresponds to the first point on a steeply rising rotation curve, it is possible that this difference is the result of an incorrect correction for beam smearing.

UGC 8837. The $\mathrm{H}$ I and the poorly sampled $\mathrm{H} \alpha$ rotation curves are in good agreement.

UGC 9211. The $\mathrm{HI}$ and $\mathrm{H} \alpha$ rotation curves are in good agreement.

UGC 10310. The $\mathrm{H} \alpha$ rotation curve falls below the $\mathrm{H}$ I rotation curve. The reason for this difference is unclear.

UGC 11557. The $\mathrm{H}$ I and $\mathrm{H} \alpha$ rotation curves agree well, but in the outer parts the $\mathrm{H} \alpha$ curve falls slightly below the H I curve.

UGC 11707. Although the $\mathrm{H} \alpha$ rotation curve is poorly sampled, the inner rise in the $\mathrm{H} \alpha$ rotation curve seems somewhat steeper than that of the H I curve.

UGC 11861. The $\mathrm{H} \alpha$ rotation curve has significant scatter, but seems to rise more steeply in the central regions. In the outer parts both curves agree well.

UGC 12732. The $\mathrm{H} \alpha$ and $\mathrm{H}$ I rotation curves are in agreement.

The detailed comparison of $\mathrm{HI}$ and $\mathrm{H} \alpha$ rotation curves shows a fairly good general agreement between the two sets, indicating that the method used to derive beam smearing corrected rotation curves is successful to a large degree. There are, however, several systematic differences. For example, the $\mathrm{H}$ I data are not sensitive to features in the central rotation curve if they are 

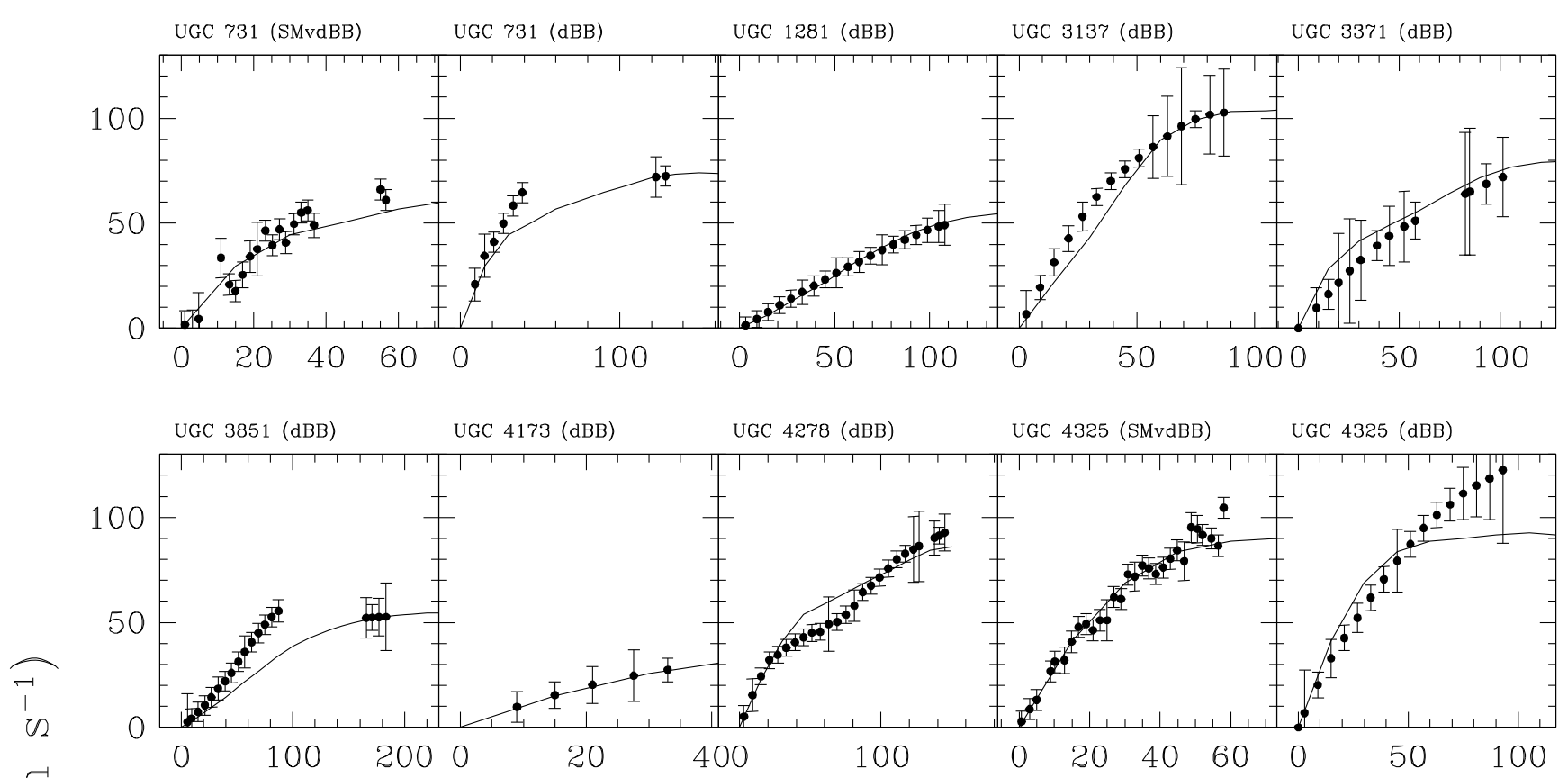

0
5
0
0
0
0
0
0
0
0
0
0
0
0
0
0
0
0
0

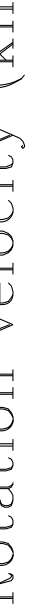
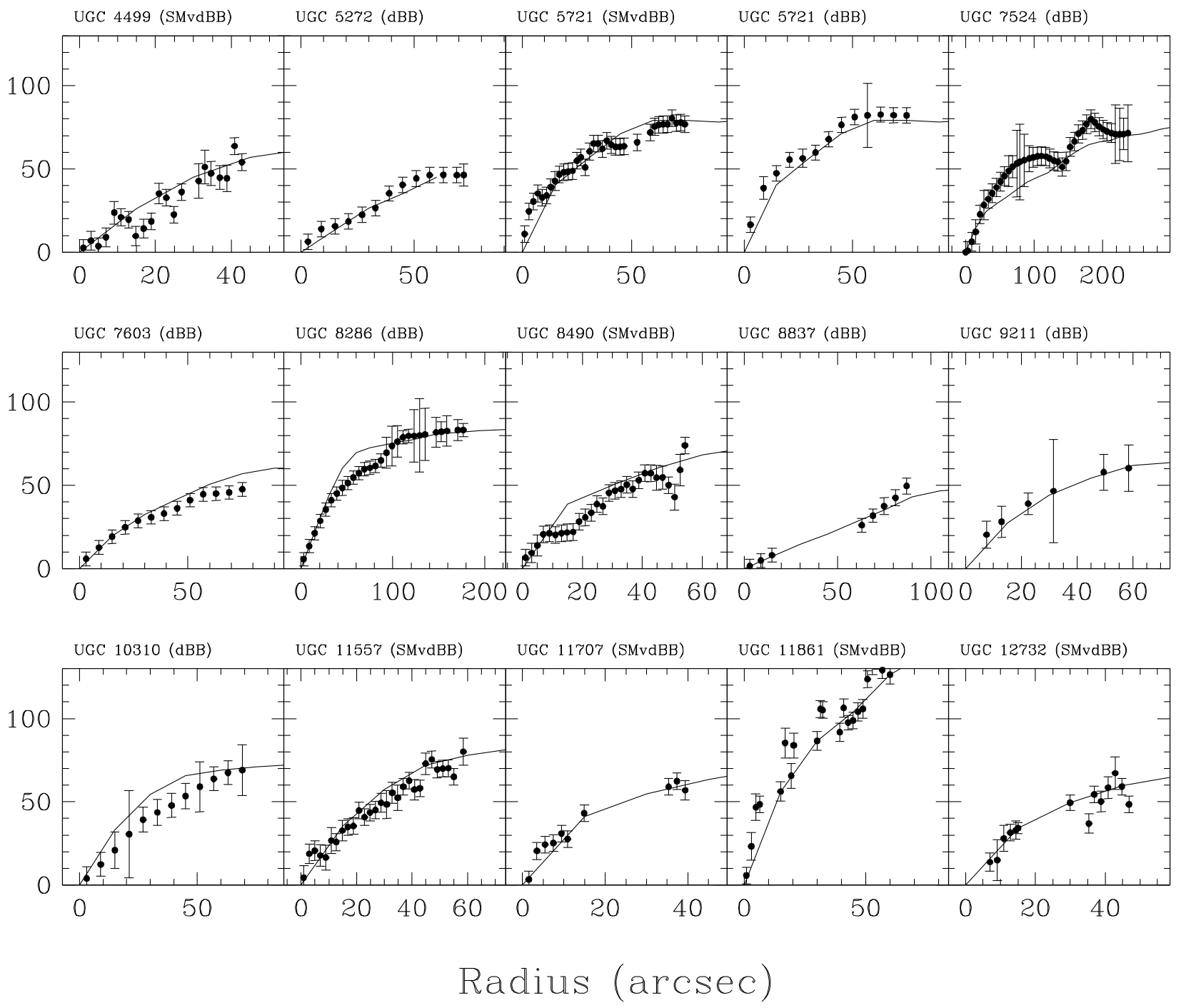

Fig. 3. Comparison of the $\mathrm{H} \alpha$ rotation curves (dots with error bars) with $\mathrm{H}$ I rotation curves (solid lines). 


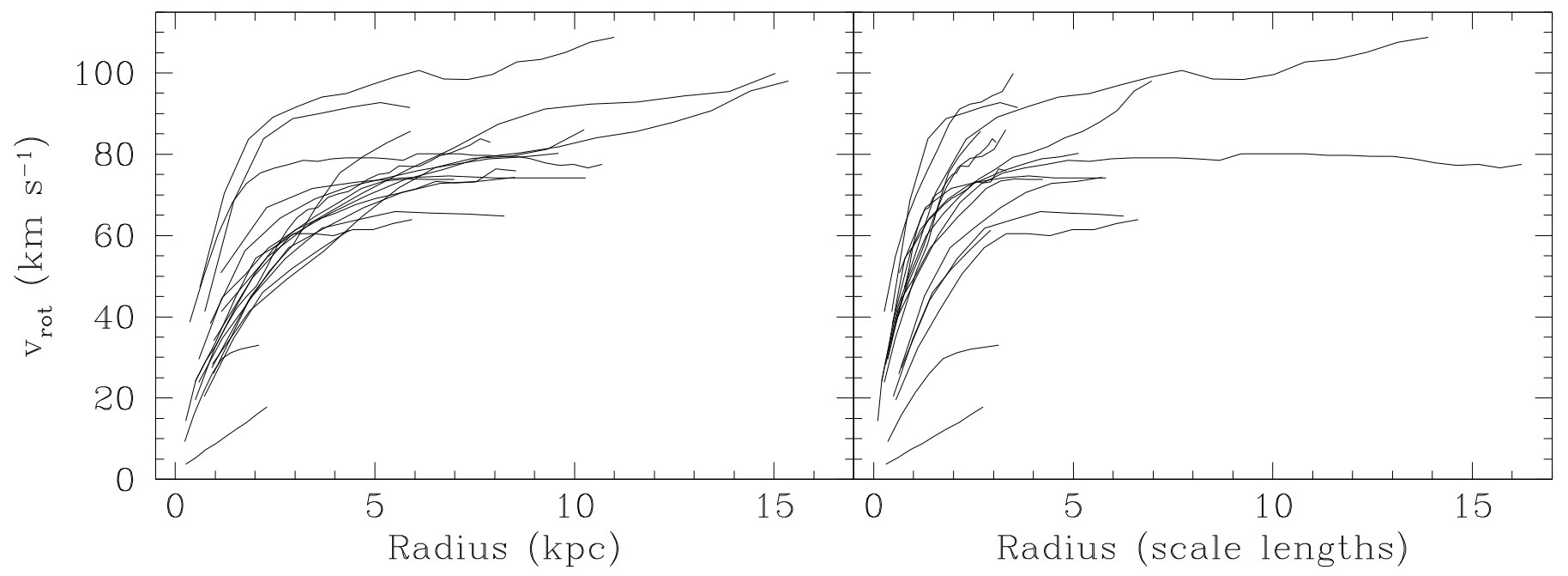

Fig. 4. Compilation of the rotation curves of the galaxies in the high quality sample. The left panel shows the rotation curves with radii expressed in $\mathrm{kpc}$, in the right panel the radii are expressed in units of disk scale lengths.

smaller than the size of the H I beam (UGC 5721, UGC 11707, UGC 11861). In addition, if the velocity gradient over the beam is large, the derived $\mathrm{HI}$ rotation curve becomes more uncertain (UGC 3137, UGC 7603, UGC 8490). On the other hand, the $\mathrm{H} \alpha$ data may be sensitive to small scale structure in the velocity field (UGC 731, UGC 3851, UGC 4325, UGC 7524), because it is derived from a $1^{\prime \prime}$ wide slice through the velocity field, whereas the H I rotation curve is derived from the velocity field as a whole. Systematic differences between the H I and the $\mathrm{H} \alpha$ rotation curves are common in edge-on galaxies (UGC 3137, UGC 4278, UGC 8286). For these galaxies, derivation of the rotation curves is difficult (see Sect. 3), especially for the $\mathrm{H} \alpha$ data, for which the typical instrumental velocity resolution of around $75 \mathrm{~km} \mathrm{~s}^{-1}$ and the possibility of extinction along the line of sight make it difficult to determine the extreme velocity accurately (see e.g. SMvdBB).

For two galaxies, UGC 3371 and UGC 10310 there were no obvious reasons for the difference between the $\mathrm{HI}$ and $\mathrm{H} \alpha$ rotation curves. The effects discussed above may all have contributed. Interestingly, Swaters et al. (2003b) have reobserved UGC 3371. Based on a high-resolution, two-dimensional $\mathrm{H} \alpha$ velocity field, they derive a rotation curve that agrees well with the $\mathrm{HI}$ rotation curve. They suggest that the differences between their rotation curve and the $\mathrm{dBB}$ rotation curve may be due to that fact that they used two-dimensional data, and the fact that dBB spline-interpolated their data.

Blais-Ouellette et al. (2001) and Garrido et al. (2002) compared rotation curves derived from Fabry-Perot imaging spectroscopy to those derived from H I synthesis observations, and found good general agreement between the $\mathrm{H} \alpha$ and $\mathrm{HI}$ rotation curves for galaxies that are well-resolved. For galaxies that are less well-resolved the relation between the $\mathrm{HI}$ and $\mathrm{H} \alpha$ shows larger scatter, leading Garrido et al. (2002) to conclude that corrections for beam smearing have introduced uncertainties. Although corrections for beam smearing may explain part of this scatter, uncertainties in the $\mathrm{H} \alpha$ Fabry-Perot data will also play a role e.g., because Fabry-Perot are less sensitive to the faint $\mathrm{H} \alpha$ emission, and hence are biased towards regions of strong star formation, which may be associated with shocks, spiral arms, and bars.

Although we argue that the effects of beam smearing on H I data can usually be corrected for the type of galaxies presented here, and that the $\mathrm{HI}$ rotation curves are therefore reliable, it is obvious that higher angular resolution observations are needed to sample the detailed rotation curve shapes in the central $30^{\prime \prime}$.

\section{Rotation curve shapes}

\subsection{The rotation curves}

For the study of the properties of late-type dwarf galaxy rotation curves, our sample has been divided into two subsamples. The high quality sample contains 19 galaxies with inclinations $39^{\circ} \leq i \leq 80^{\circ}$ and rotation curves with quality $q=1$. A lower limit was applied because at low inclinations the rotation curve amplitude and shape are uncertain due to higher inclination uncertainties. The particular value of $39^{\circ}$ was chosen so that UGC 12732 is included in the sample. UGC 11861 was excluded from the analysis below because of its high luminosity, resulting in a high quality sample of 18 galaxies. The lower quality sample contains 16 galaxies with inclinations $39^{\circ} \leq i \leq 80^{\circ}$ and rotation curves with $q=2$. UGC 4543 was excluded because of its high luminosity. The lower quality sample thus selected contains 15 dwarf galaxies.

In Fig. 4 the rotation curves of the galaxies in the high quality sample are shown. The left panel shows the rotation curves with the radii expressed in $\mathrm{kpc}$, in the right panel the radii are expressed in units of optical disk scale lengths. A striking feature of Fig. 4 is that the rotation curves of the dwarf galaxies in our sample look similar to those of spiral galaxies, in particular when the radii are expressed in units of disk scale lengths: most of the rotation curves rise rapidly in the inner parts and start to flatten after about two disk scale lengths. Although about half of the galaxies in our high-quality sample do not extend significantly beyond three disk scale lengths, those that do have rotation curves that are fairly flat, including amplitudes as low as $60 \mathrm{~km} \mathrm{~s}^{-1}$. Thus, it seems that whether the flat part of a rotation curve is reached or not depends more on the radial extent of the rotation curve than on its amplitude. None of the galaxies in our sample have declining rotation curves, even though some of the rotation curves extend to beyond 10 disk scale lengths.

To compare the properties of the rotation curves of the late-type dwarf galaxies in our sample to the rotation curve properties of other galaxies, we have constructed a comparison 


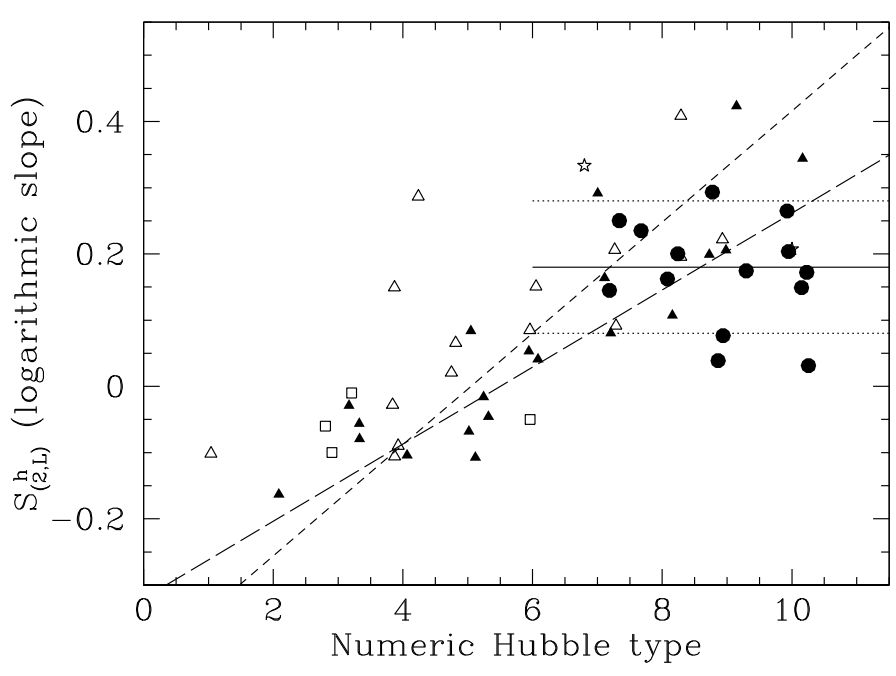

Fig. 5. Logarithmic slope of the rotation curve between 2 disk scale lengths and the last measured point $S_{(2, \mathrm{~L})}^{h}$ versus the numeric Hubble type. Only galaxies for which the last measured point lies beyond 3 disk scale lengths and with inclinations between $39^{\circ}$ and $80^{\circ}$ have been plotted. A random value between -0.5 and 0.5 has been added to the numeric Hubble type to spread the points in the plots. The filled circles represent the late-type galaxies with high quality rotation curves from the sample presented in this paper. The open triangles represent galaxies from the Ursa Major sample (Verheijen 1997; Verheijen \& Sancisi 2001), filled triangles represent the galaxies from various sources presented in Broeils (1992a), open squares the galaxies from Spekkens \& Giovanelli (2006). The stars represent galaxies with $\mathrm{H} \alpha$ rotation curves from the samples of de Blok \& Bosma (2002) and Swaters et al. (2003a). The long-dashed line represents the relation between Hubble and rotation curve shape as reported by Casertano \& van Gorkom (1991), the short-dashed line the same relation as found by Broeils (1992a). The horizontal solid and dotted lines indicate the average value for $S_{(2, \mathrm{~L})}^{h}$ and the $1 \sigma$ range for galaxies with Hubble type later than Sc (see text for details).

sample of extended $\mathrm{H}$ I rotation curves. We used the $\mathrm{H}$ I rotation curves of Ursa Major galaxies by Verheijen (1997) and Verheijen \& Sancisi (2001). This sample spans a range of galaxy types, but has relatively few early-type galaxies and no galaxies with $M_{R}>-17$. We only included galaxies with at least 5 measured points in the rotation curve to ensure sufficient resolution. We also included the sample presented in Broeils (1992a), which consists of a collection of rotation curves presented in the literature. It includes a large range of galaxy types and luminosities, spanning from $M_{R}=-23$ to $M_{R}=-15$. Most of the photometric data presented in Broeils (1992a) are in the $B$-band. These were converted to $R$-band assuming $B-R=1.0$. A third source is the study by Spekkens \& Giovanelli (2006), who have studied a sample of massive late-type spiral galaxies (their average Hubble type is Sbc). We assumed $R-I=0.5$ to convert their $I$-band photometry to the $R$-band. For each of these literature samples we only used galaxies with inclinations $39^{\circ} \leq i \leq 80^{\circ}$. The combined literature sample will be used as comparison in the following discussion.

\subsection{Rotation curve shape and morphology}

The relation between rotation curve shape and morphology is explored in Fig. 5. In this figure, the logarithmic slope between two disk scale lengths and the last measured point, $S_{(2, \mathrm{~L})}^{h}$, is plotted against the numeric Hubble type. Only galaxies in which the H I extends to beyond three disk scale lengths have been plotted. For galaxies with less extended H I disks $S_{(2, \mathrm{~L})}^{h}$ is not well-determined because the rotation velocities lie on the rising branch. In Fig. 5, the filled circles represent the highquality sample. Also shown in these figures are the logarithmic slopes for the spiral galaxies in the samples of Verheijen \& Sancisi (2001, open triangles), Broeils (1992a, filled triangles), and Spekkens \& Giovanelli (2006, open squares). We have also plotted the logarithmic slopes for the galaxies with $\mathrm{H} \alpha$ rotation curves, from the samples of $\mathrm{dBB}$ and SMvdBB (stars).

We have chosen to use $S_{(2, \mathrm{~L})}^{h}$ rather than the slope between two thirds of the optical radius $R_{25}$ and the last measured point (as was done by e.g., CvG and Broeils 1992a). Both choices have their merits: using disk scale lengths, the shape parameter is measured at the same relative locations in the disks, and using the optical radius the shape is measured at the same surface brightness. We have also used the optical radius to define the shape parameter, and found that the results hardly depend on the choice of the scaling parameter, as was also found by $\mathrm{CvG}$.

Both CvG and Broeils (1992a) have reported tight correlations between the shape of the rotation curves and the Hubble type. These correlations are plotted for reference in Fig. 5.

With the inclusion of the new data reported here, it now appears that galaxies with Hubble types later than Sc all have similar outer rotation curve slopes, with an average $S_{(2, \mathrm{~L})}^{h}$ of $0.18 \pm 0.10$ (marked in Fig. 5 by the solid and dotted lines). None of the galaxies in our high-quality sample have rotation curves as shallow as seen among the dwarf galaxies in the comparison samples. We will discuss this in Sect. 7.

\subsection{Rotation curve shape, amplitude, and absolute magnitude}

To investigate the relation between the rotation curve shape, amplitude, and absolute magnitude, we have plotted the rotation curves grouped into six intervals in absolute magnitude in Fig. 6. In this figure, the radii are expressed in units of disk scale length. The full lines refer to galaxies in the high quality sample and the dotted lines to galaxies in the lower quality sample. The most obvious relation is the decrease in amplitude towards fainter absolute magnitudes, as expected from the Tully-Fisher relation. Note that there is a spread in rotation curve shapes in each luminosity bin. This spread may in part be observational in nature, e.g., as a result of inclination errors and asymmetries in the galaxies. The top left panel contains late-type galaxies in the transition regime between bright galaxies and dwarf galaxies.

The relations between absolute magnitude or maximum rotation velocity on the one hand, and rotation curve shape on the other, are made more explicit in Figs. 7 (top panel) and 8.

At first sight, the correlation seen between the rotation curve shape $S_{(2, \mathrm{~L})}^{h}$ and absolute magnitude (see Fig. 7, top panel) seems to confirm the results found by e.g., Rubin et al. (1985), Persic \& Salucci (1988), Broeils (1992a), and CvG that rotation curves rise more slowly towards lower luminosities. However, with the addition of the dwarf galaxies presented here, the dwarf regime is better sampled and extended down to fainter absolute magnitudes, thus making it possible to sample this correlation in more detail at the low luminosity end.

In Fig. 5 it was shown that the rotation curve shape $S_{(2, \mathrm{~L})}^{h}$ is similar for galaxies with Hubble types later than Sc. A similar trend is seen with absolute magnitude, as can be seen in the top panel of Fig. 7. This is not unexpected, given the tight correlation between Hubble type and absolute magnitude (bottom 
Table 3. Optical and rotation curve properties of the comparison sample.

\begin{tabular}{|c|c|c|c|c|c|c|c|c|c|c|c|c|c|c|c|}
\hline Name & Ref. & $\begin{array}{r}D_{a} \\
\mathrm{Mpc} \\
(3)\end{array}$ & $\begin{array}{r}M_{R} \\
\mathrm{mag} \\
(4)\end{array}$ & Type & $\begin{array}{r}h \\
\mathrm{kpc} \\
(6)\end{array}$ & $\begin{array}{r}\mu_{0}^{R} \\
\mathrm{mag}^{\prime \prime-2} \\
\text { (7) }\end{array}$ & $\begin{array}{l}v_{1} \\
- \\
(8)\end{array}$ & $\begin{array}{c}v_{2} \\
--- \\
(9)\end{array}$ & $\begin{array}{c}v_{3} \\
\mathrm{~km} \mathrm{~s}^{-1} \\
(10)\end{array}$ & $\begin{array}{r}v_{4} \\
-\quad- \\
(11)\end{array}$ & $\begin{array}{c}v_{\text {last }} \\
-\quad- \\
(12)\end{array}$ & $\begin{array}{l}r_{\text {last }} \\
\mathrm{kpc} \\
(13)\end{array}$ & $S_{(1,2)}$ & $S_{(2,3)}$ & $S_{(2, \mathrm{~L})}$ \\
\hline NGC 55 & B & 1.6 & -19.6 & .SBS9* & 1.6 & 20.5 & 47 & 73 & 85 & 86 & 87 & 7.7 & 0.63 & 0.40 & 0.20 \\
\hline NGC 247 & B & 2.5 & -19.0 & .SXS7.. & 2.9 & 22.4 & 77 & 104 & - & - & 108 & 7.4 & 0.43 & - & - \\
\hline NGC 300 & B & 1.8 & -18.8 & .SAS7.. & 2.1 & 21.2 & 72 & 89 & 97 & - & 93 & 8.0 & 0.30 & 0.22 & 0.08 \\
\hline NGC 801 & B & 79.2 & -22.7 & $. S . .5 .$. & 12 & 20.9 & 223 & 222 & 214 & - & 216 & 44 & -0.01 & -0.09 & -0.05 \\
\hline UGC 2259 & B & 9.8 & -18.0 & .SBS8.. & 1.3 & 21.3 & 70 & 83 & 85 & 88 & 90 & 5.7 & 0.25 & 0.06 & 0.11 \\
\hline NGC 1324 & SG & 79 & -23.1 & $. S . .3 *$ & 5.6 & 18.9 & 258 & 282 & 300 & 299 & 264 & 35 & 0.13 & 0.15 & -0.06 \\
\hline UGC 2849 & SG & 116 & -23.0 & $. S . .6 \%$ & 6.2 & 20.1 & 245 & 277 & 280 & 277 & 267 & 34 & 0.18 & 0.03 & -0.04 \\
\hline UGC 2885 & B & 78.7 & -23.8 & .SAT5.. & 13 & 21.0 & 275 & 280 & 296 & 298 & 298 & 54 & 0.03 & 0.14 & 0.08 \\
\hline NGC 1560 & B & 3 & -16.9 & SAS7. & 1.3 & 22.2 & 42 & 59 & 65 & 74 & 77 & 6.2 & 0.49 & 0.24 & 0.29 \\
\hline UGC 3371 & $\mathrm{dBB}$ & 12.8 & -17.74 & .I...9*. & 3.09 & 23.3 & 47 & 71 & - & - & 72 & 6.3 & 0.60 & - & - \\
\hline NGC 2403 & B & 3.3 & -20.3 & .SXS6.. & 2.1 & 20.4 & 102 & 127 & 128 & 131 & 134 & 14.9 & 0.31 & 0.02 & 0.04 \\
\hline NGC 2841 & B & 18 & -22.7 & .SAR3*. & 4.6 & 20.1 & 322 & 327 & 319 & 298 & 294 & 61 & 0.02 & -0.06 & -0.06 \\
\hline NGC 2903 & B & 6.4 & -21.0 & .SXT4.. & 2.0 & 19.5 & 209 & 211 & 202 & 198 & 180 & 18.2 & 0.01 & -0.11 & -0.10 \\
\hline NGC 2955 & SG & 105 & -22.8 & PSAR3.. & 4.9 & 19.4 & 244 & 244 & 262 & 241 & 208 & 47 & 0.00 & 0.18 & -0.10 \\
\hline NGC 2998 & B & 67.4 & -22.9 & .SXT5.. & 5.4 & 19.3 & 206 & 215 & 213 & 214 & 198 & 35 & 0.06 & -0.02 & -0.07 \\
\hline UGC 5272 & $\mathrm{dBB}$ & 6.1 & -15.11 & .I..9.. & 0.60 & 22.4 & 22 & 40 & 47 & - & 46 & 2.1 & 0.87 & 0.37 & 0.21 \\
\hline NGC 3109 & B & 1.7 & -17.8 & .SBS9. & 1.6 & 22.2 & 31 & 50 & 61 & - & 66 & 6.2 & 0.67 & 0.51 & 0.42 \\
\hline NGC 3198 & B & 9.4 & -20.4 & .SBT5.. & 2.6 & 20.6 & 135 & 153 & 155 & 154 & 149 & 22.6 & 0.18 & 0.03 & -0.02 \\
\hline UGC 5721 & SMBB & 6.7 & -16.58 &.$S X .7 ?$ & 0.45 & 20.2 & 41 & 57 & 64 & 68 & 77 & 2.42 & 0.47 & 0.29 & 0.33 \\
\hline UGC 6399 & VS & 15.5 & -18.3 & $. S . .9 *$. & 1.8 & 22.2 & 53 & 76 & 84 & - & 88 & 6.8 & 0.52 & 0.25 & 0.22 \\
\hline UGC 6446 & VS & 15.5 & -18.5 & SA.7.. & 2.6 & 22.2 & 62 & 74 & 81 & 85 & 80 & 13.2 & 0.24 & 0.23 & 0.09 \\
\hline NGC 3726 & VS & 15.5 & -21.3 & .SXR5.. & 4.4 & 20.1 & 124 & 163 & 150 & 150 & 167 & 28 & 0.40 & -0.21 & 0.02 \\
\hline NGC 3877 & VS & 15.5 & -21.2 & .SAS5*. & 2.5 & 19.1 & 131 & 161 & 171 & - & 169 & 9.8 & 0.31 & 0.14 & 0.07 \\
\hline NGC 3917 & VS & 15.5 & -20.4 & .SA.6*. & 2.4 & 20.9 & 80 & 119 & 136 & 137 & 137 & 12.8 & 0.57 & 0.33 & 0.15 \\
\hline NGC 3949 & VS & 15.5 & -20.6 & .SAS4*. & 1.4 & 19.2 & 118 & 151 & 160 & 167 & 169 & 6.1 & 0.36 & 0.14 & 0.15 \\
\hline NGC 3953 & VS & 15.5 & -21.7 & .SBR4.. & 3.2 & 19.5 & 193 & 220 & 225 & 239 & 215 & 13.5 & 0.19 & 0.05 & -0.03 \\
\hline NGC 3972 & VS & 15.5 & -19.8 & .SAS4.. & 1.7 & 19.9 & 79 & 107 & 122 & 135 & 134 & 7.5 & 0.44 & 0.31 & 0.29 \\
\hline UGC 6917 & VS & 15.5 & -19.2 & .SB.9.. & 2.8 & 21.9 & 81 & 101 & 111 & - & 111 & 9.0 & 0.32 & 0.23 & 0.21 \\
\hline NGC 3985 & VS & 15.5 & -19.0 & .SBS9*. & 0.8 & 19.5 & 42 & 73 & - & - & 93 & 2.3 & 0.82 & - & - \\
\hline UGC 6923 & VS & 15.5 & -18.4 & .I..9*. & 1.3 & 21.5 & 46 & 72 & 79 & - & 81 & 4.6 & 0.66 & 0.23 & 0.20 \\
\hline NGC 3992 & VS & 15.5 & -21.8 & .SBT4.. & 3.4 & 19.2 & - & 271 & 264 & 273 & 237 & 30 & - & -0.06 & -0.09 \\
\hline UGC 6983 & VS & 15.5 & -19.0 & .SBT6.. & 3.0 & 22.0 & 92 & 102 & 113 & 108 & 109 & 13.5 & 0.14 & 0.26 & 0.085 \\
\hline UGC 7089 & VS & 15.5 & -19.1 & $. S . .8 *$ & 2.5 & 22.5 & 45 & 65 & 77 & - & 79 & 7.9 & 0.53 & 0.41 & 0.41 \\
\hline NGC 4100 & VS & 15.5 & -21.0 & PSAT4.. & 2.4 & 19.2 & 125 & 185 & 195 & 192 & 159 & 19.6 & 0.56 & 0.12 & -0.11 \\
\hline NGC 4138 & VS & 15.5 & -20.6 & $. \mathrm{LAR}+.$. & 1.2 & 19.1 & 168 & 181 & 192 & 195 & 150 & 16.0 & 0.11 & 0.15 & -0.10 \\
\hline NGC 4218 & VS & 15.5 & -18.5 & $. S . .1 \$$. & 0.7 & 19.8 & 83 & 104 & - & - & 73 & 1.5 & 0.34 & - & - \\
\hline UGC 7603 & dBB & 6.8 & -16.88 & .SBS7? & 0.90 & 20.8 & 29 & 43 & - & - & 48 & 2.5 & 0.59 & - & - \\
\hline DDO 154 & B & 4 & -14.8 & .IBS9.. & 0.5 & 22.2 & 15 & 24 & 32 & 37 & 43 & 5.7 & 0.64 & 0.70 & 0.34 \\
\hline NGC 5033 & B & 11.9 & -21.2 & .SAS5.. & 5.8 & 20.6 & 220 & 219 & 208 & 192 & 200 & 27 & -0.01 & -0.12 & -0.11 \\
\hline DDO 168 & B & 3.5 & -16.2 & .IB.9.. & 0.9 & 22.4 & 31 & 51 & - & - & 49 & 2.5 & 0.71 & - & - \\
\hline DDO 170 & B & 12 & -15.5 & .I.. $9 *$ & 1.3 & - & 32 & 50 & 56 & 59 & 62 & 7.2 & 0.67 & 0.27 & 0.21 \\
\hline NGC 5533 & B & 55.8 & -22.4 & .SAT2.. & 11.4 & 21.0 & 274 & 263 & 240 & 245 & 227 & 56 & -0.06 & -0.22 & -0.16 \\
\hline NGC 5585 & B & 6.2 & -18.5 & $. S X S 7 .$. & 1.4 & 20.9 & 54 & 77 & 89 & 92 & 89 & 7.2 & 0.49 & 0.37 & 0.16 \\
\hline UGC 9211 & dBB & 12.6 & -16.21 &. .I.. $9 *$. & 1.32 & 22.6 & 38 & 55 & - & - & 60 & 3.6 & 0.52 & - & - \\
\hline NGC 6195 & SG & 131 & -23.2 & .S..3.. & 6.1 & 19.9 & 236 & 245 & 247 & 250 & 243 & 40 & 0.05 & 0.02 & -0.01 \\
\hline NGC 6503 & B & 5.9 & -19.7 & .SAS6.. & 1.7 & 20.9 & 96 & 113 & 114 & 115 & 122 & 15.1 & 0.23 & 0.03 & 0.05 \\
\hline NGC 6674 & B & 49.3 & -22.6 & .SBR3.. & 8.3 & 21.5 & 290 & 265 & 247 & 235 & 242 & 52 & -0.13 & -0.17 & -0.08 \\
\hline NGC 7331 & B & 14.9 & -22.4 & .SAS3.. & 4.5 & 20.5 & 257 & 246 & 233 & 238 & 238 & 28 & -0.06 & -0.13 & -0.03 \\
\hline
\end{tabular}

(1) Galaxy name (ordered by RA); (2) source of the rotation curve data. B represents Broeils (1992), dBB is de Blok \& Bosma (2002), SG means Spekkens \& Giovanelli (2006), SMBB is Swaters et al. (2003a), and VS means Verheijen \& Sancisi (2001); (3) the adopted distance, taken from the original papers; (4) the absolute $R$-band magnitude; (5) the morphological type according to the RC3 catalog (de Vaucouleurs et al. 1991), using the same coding; (6) and (7) the disk scale length and the $R$-band central disk surface brightness. Optical data are from Paper II for the dBB and SMBB data, from Verheijen (1997) for the VS data, from Spekkens \& Giovanelli (2006) for the SG data, and from Broeils (1992) for the B data. The data from Broeils (1992) are in the $B$-band. These were converted to $R$-band assuming $B-R=1.0$. (8) to (11) the rotation velocities at one, two, three and four disk scale lengths; (12) and (13) the rotation velocity at the last measured point and the radius of the last measured point; (14), (15) and (16) the logarithmic shape of the rotation curve between one and two disk scale lengths, between two and three disk scale lengths, and between two disk scale lengths and the last measured point. 


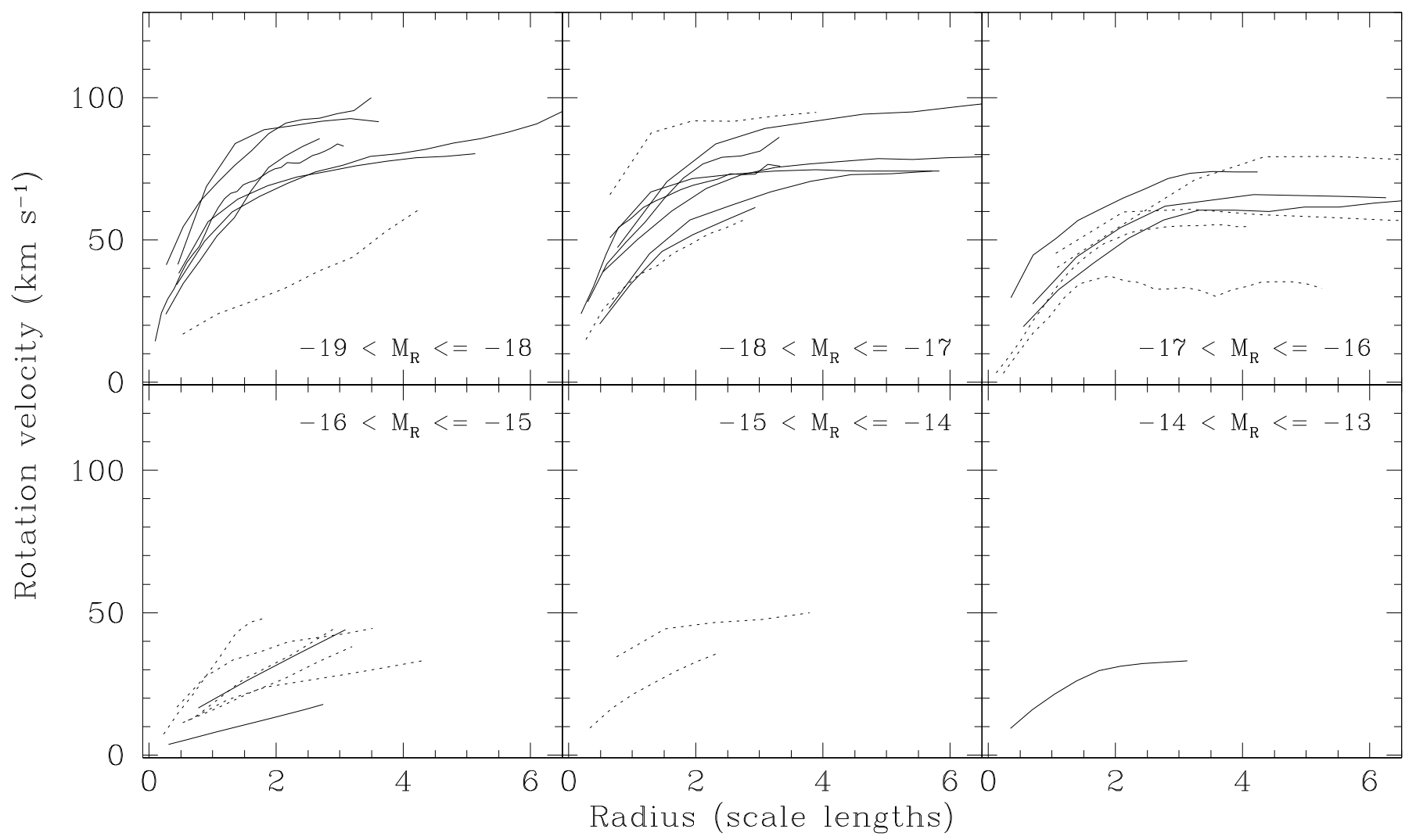

Fig. 6. Rotation curves in bins of absolute magnitude with radii expressed in units of disk scale length. The full lines give the rotation curves of the galaxies in the high quality sample, the dotted lines those of the galaxies in the lower quality sample.

panel of Fig. 7). From Fig. 7 it is clear that, down to the faintest magnitudes, all the galaxies in our sample with high quality rotation curves have logarithmic slopes between 0 and 0.3 , although the scatter may increase somewhat towards lower absolute magnitudes. For these galaxies, the mean value of $S_{(2, \mathrm{~L})}^{h}$ is $0.17 \pm 0.08$, where the uncertainty is the dispersion around the mean. The logarithmic slopes of late-type spiral galaxies with $-20.5<M_{R}<-18$ have similar values. For these galaxies, the average $S_{(2, \mathrm{~L})}^{h}$ is $0.15 \pm 0.11$. Thus, the results in Fig. 7 indicate that the outer rotation curve shapes, as measured by $S_{(2, \mathrm{~L})}^{h}$, are similar for late-type spiral galaxies and the late-type dwarf galaxies in our sample. Therefore, for galaxies fainter than $M_{R}=-20$, the outer rotation curve shape does not appear to depend on luminosity down to $M_{R} \sim-16$ and possibly even $M_{R} \sim-14$, although we have little information at the lowest luminosities.

At the brighter end, as was already pointed out by $\mathrm{CvG}$ and Broeils (1992a), the most luminous galaxies tend to have declining rotation curves. This is also seen in Fig. 7. Galaxies brighter than about $M_{R} \sim-21$ mostly have negative values of $S_{(2, \mathrm{~L})}^{h}$, indicating lower rotation velocities at the last measured points. Virtually all of these galaxies have bulges, as is demonstrated in the bottom panel of Fig. 7, where the $S_{(2, \mathrm{~L})}^{h}$ is plotted against the numerical Hubble type. A larger sample of early-type spiral and high-luminosity galaxies is needed to study the details of the correlations between rotation curve shape and morphology or luminosity.

Following $\mathrm{CvG}$, we have also plotted $S_{(2, \mathrm{~L})}^{h}$ against the maximum rotation velocity in Fig. 8. The relation between these quantities found by $\mathrm{CvG}$, represented by the dotted line, is given for comparison. The galaxies in the sample presented here follow the same relation. This may seem to indicate that the results presented here are in good agreement with the relation found by $\mathrm{CvG}$. However, at the lowest rotation velocities, the galaxies in the comparison sample tend to fall above the $\mathrm{CvG}$ relation. In fact, 4 out 5 galaxies with $v_{\max }<80 \mathrm{~km} \mathrm{~s}^{-1}$ fall well above the dotted line. Similarly, the slowest rotators in Fig. 6 of $\mathrm{CvG}$ also fall above their relation. Thus, even though the values for $S_{(2, \mathrm{~L})}^{h}$ for the galaxies in our sample are consistent with the $\mathrm{CvG}$ relation, the $S_{(2, \mathrm{~L})}^{h}$ values for our high-quality sample are significantly different from those for our reference sample. We point out, however, that there are only a small number of galaxies with $v_{\max }<80 \mathrm{~km} \mathrm{~s}^{-1}$. To fully sample the relation between rotation curve shape and $v_{\max }$, a larger number of beamsmearing corrected rotation curves is needed. We also note that at the higher mass end the galaxies in our sample fall above the CvG relation. This was also noted by Broeils (1992a), who remarked that there is a clear correlation between the slope and the maximum velocity, but that it is not a simple linear relation.

The slope of the rotation curve is weakly correlated with surface brightness, as can be seen in Fig. 9. At intermediate surface brightnesses, there is a large spread in slopes. At the high surface brightness end, galaxies tend to have flat or declining rotation curves, and at the low surface brightness end, galaxies tend to have slowly rising rotation curves.

\subsection{Rotation curve shape and light distribution}

To investigate a possible link between the light distribution and the rotation curve, we compare the central concentration of light and the inner rotation curve shape. A straightforward measure of 


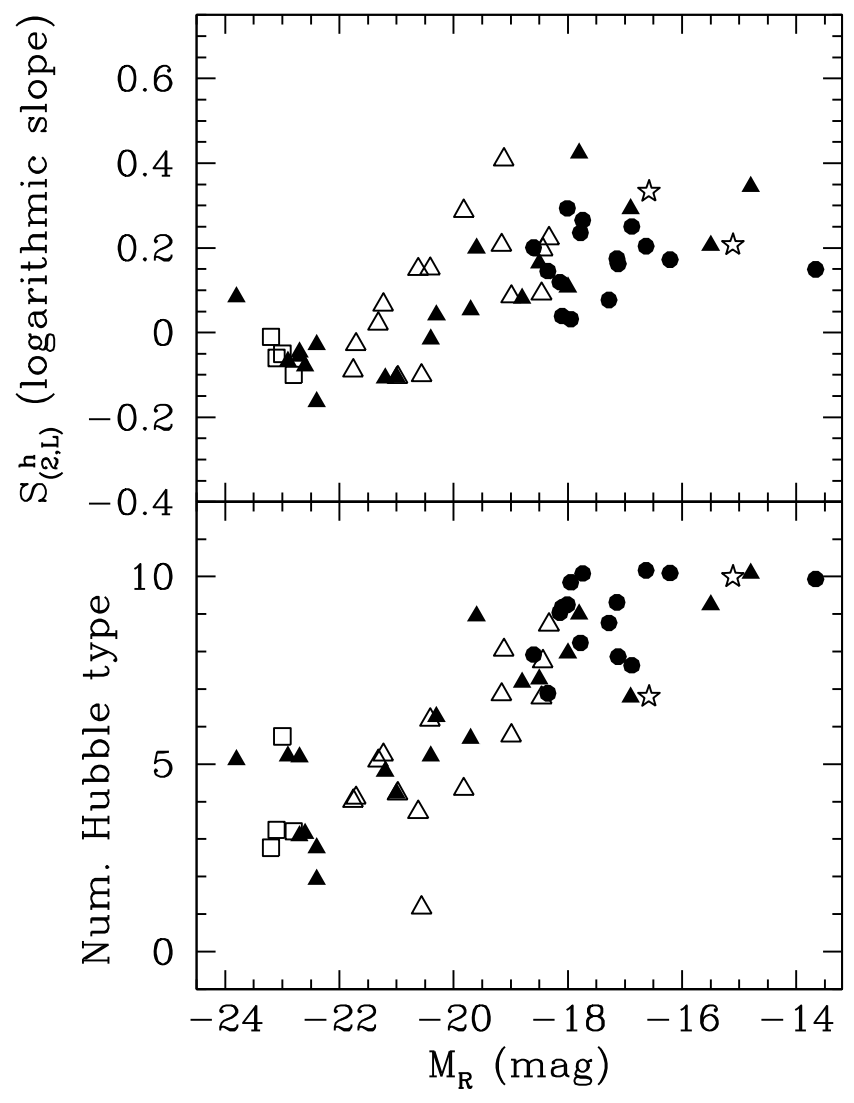

Fig. 7. Logarithmic slope of the rotation curve between 2 disk scale lengths and the last measured point $S_{(2, \mathrm{~L})}^{h}$ versus the the absolute $R$-band magnitude $M_{R}$ (top), and numeric Hubble type versus $M_{R}$ (bottom). Only galaxies for which the last measured point lies beyond 3 disk scale lengths and with inclinations between $39^{\circ}$ and $80^{\circ}$ have been plotted. A random value between -0.5 and 0.5 has been added to the numeric Hubble type to spread the points in the plots. Symbol coding as in Fig. 5.

the central concentration of light is given by the parameter $\Delta \mu_{R}$, which we defined as:

$\Delta \mu_{R}=\mu_{0}^{R}-\mu_{\mathrm{c}}^{R}$,

where $\mu_{\mathrm{c}}^{R}$ is the observed central surface brightness. If a bulge or a central concentration of light is present, $\Delta \mu_{R}$ is positive, and if the light profile is flat-topped, $\Delta \mu_{R}$ is negative.

A significant fraction of the late-type dwarf galaxies in our sample have non-exponential light profiles in the inner regions. Some have a central concentration of light, and in others the light profile in the center falls below that of an exponential fit to the outer parts of the light profile, making the light profile flattopped. The galaxies in the comparison samples of Verheijen \& Sancisi (2001) and Broeils (1992a) include both galaxies with strong bulges and galaxies that are dominated by exponential profiles. The galaxies in the sample of Spekkens \& Giovanelli (2006) all have bulges or central concentrations of light.

In Fig. 10, $\Delta \mu_{R}$ is plotted against $S_{(1,2)}^{h}$, the logarithmic slope between one and two disk scale lengths, for the late-type dwarf galaxies in our sample and for the galaxies in the comparison samples. A clear correlation is found between $\Delta \mu_{R}$ and $S_{(1,2)}^{h}$. Rotation curves of galaxies with a central concentration of light are relatively flat between one and two disk scale lengths, and therefore they must rise steeply within one disk scale length. Galaxies with a flat-topped light profile have rotation curves

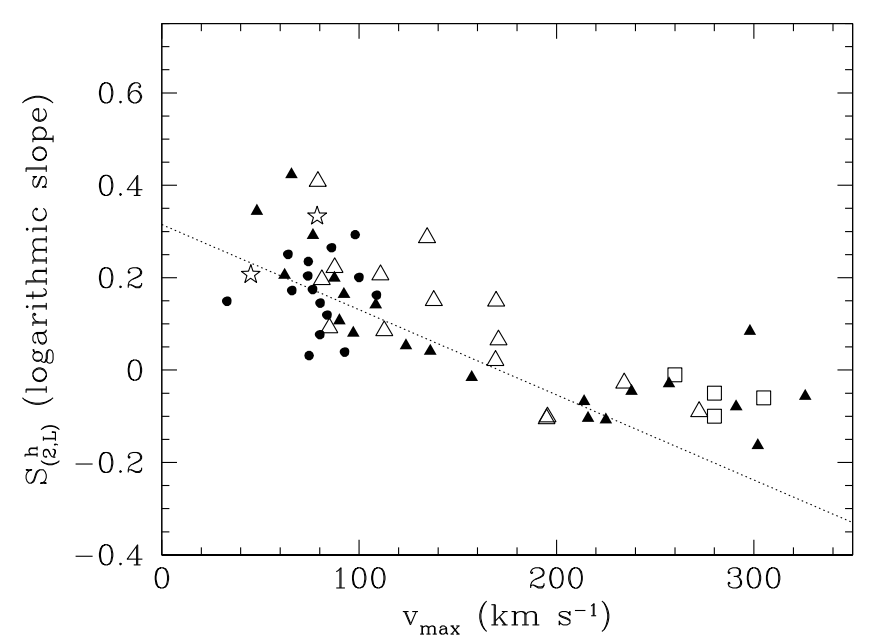

Fig. 8. Logarithmic slope of the rotation curve between 2 disk scale lengths and the last measured point $S_{(2, \mathrm{~L})}^{h}$ versus the maximum rotation velocity. Only galaxies for which the last measured point lies beyond 3 disk scale lengths and with inclinations between $39^{\circ}$ and $80^{\circ}$ have been plotted. Symbol coding as in Fig. 5. The dotted line represents the correlation between rotation curve shape and maximum rotation velocity found by Casertano \& van Gorkom (1991).

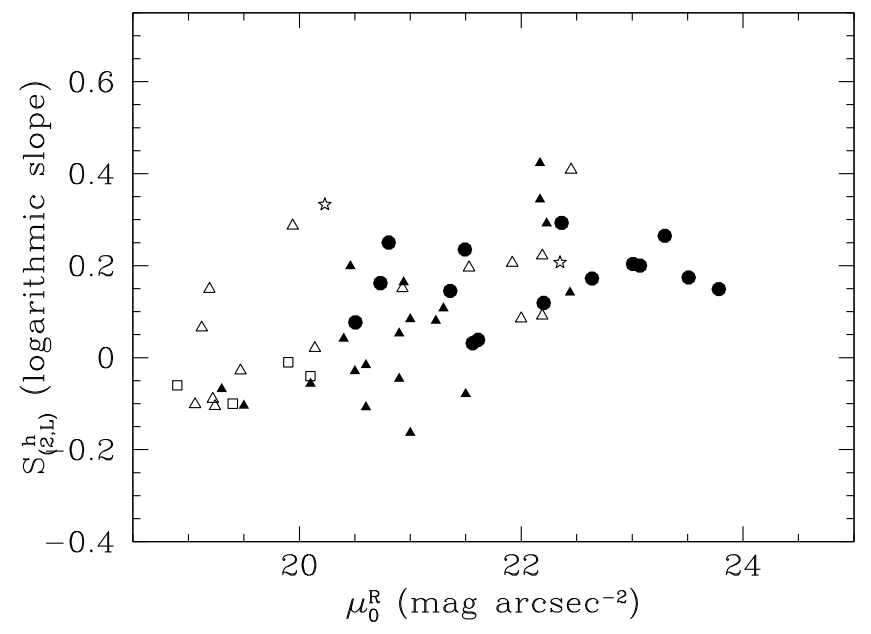

Fig. 9. Logarithmic slope of the rotation curve between 2 disk scale lengths and the last measured point $S_{(2, \mathrm{~L})}^{h}$ versus the central extrapolated disk surface brightness $\mu_{0}^{R}$. Only galaxies for which the last measured point lies beyond 3 disk scale lengths and with inclinations between $39^{\circ}$ and $80^{\circ}$ have been plotted. Symbol coding as in Fig. 5 .

that rise more slowly in the inner parts. This correlation between $\Delta \mu_{R}$ and $S_{(1,2)}^{h}$ remains even if only LSB galaxies with $\mu_{R}>22 \mathrm{mag} \operatorname{arcsec}^{-2}$ are considered, although the range in $\Delta \mu_{R}$ is reduced to $-1<\Delta \mu_{R}<1$ for those galaxies. As can be seen in Fig. 10, the correlation between $\Delta \mu_{R}$ and $S_{(1,2)}^{h}$ shows considerable scatter. It is likely this is partly due to observational uncertainties, given that for two thirds of the galaxies the resolution of the H I observations is similar to the scale lengths of these galaxies.

\section{Discussion and conclusions}

In this paper, we have presented rotation curves derived from H I observations for a sample of 62 galaxies. These rotation curves have been derived by interactively fitting model data 


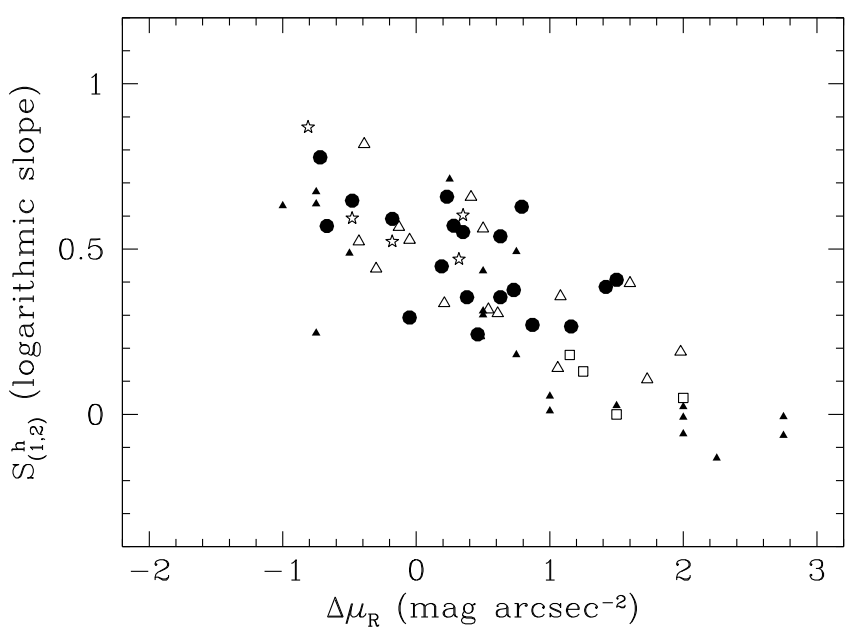

Fig. 10. Logarithmic slope of the rotation curve between 1 and 2 disk scale lengths $S_{(1,2)}^{h}$ versus the difference between extrapolated disk surface brightness $\mu_{0}^{R}$ and the observed central surface brightness $\mu_{c}^{R}$. Only galaxies with inclinations between $39^{\circ}$ and $80^{\circ}$ have been plotted. Symbol coding as in Fig. 5.

cubes to the observed cubes. This procedure takes the rotation curve shape, the H I distribution, the inclination, and the size of the beam into account, and makes it possible to correct for the effects of beam smearing. Comparison of the derived rotation curves with high resolution $\mathrm{H} \alpha$ rotation curves shows good general agreement, indicating that the scheme used to correct the rotation curves for beam smearing works well.

From our sample of 62 galaxies, we selected 18 systems with rotation curves of high quality. These have been used to study the shapes and amplitudes of the rotation curves and their relation with the distribution of light.

We have found that the rotation curve shapes of late-type dwarf galaxies in our sample are similar to those of higher luminosity, late-type spiral galaxies, in the sense that for most of the dwarf galaxies in our sample the rotation curves, when expressed in units of disk scale lengths, rise as steeply in the inner parts and start to flatten beyond about two disk scale lengths. Moreover, there are several galaxies with relatively flat rotation curves, and with amplitudes as low as $60 \mathrm{~km} \mathrm{~s}^{-1}$.

We found no galaxies with pure solid body rotation curves that extend beyond 3 disk scale lengths. Thus, whether the rotation curve becomes flat to depends mainly on the extent of the rotation curve, independent of its amplitude.

This similarity between the rotation curve shapes of late-type dwarf and late-type spiral galaxies is also borne out by the similar logarithmic slopes between two disk scale lengths and the last measured point. For the late-type dwarf galaxies in our highquality sample, $S_{(2, \mathrm{~L})}^{h}=0.17 \pm 0.08$, and for the spiral galaxies in our reference sample $S_{(2, \mathrm{~L})}^{h}=0.15 \pm 0.11$. The similarity between the rotation curve shapes of dwarf and spiral galaxies continues down to $M_{R} \sim-16 \mathrm{mag}$, and possibly even down to $M_{R} \sim-14 \mathrm{mag}$, although we have little information at the faintest luminosities. The ongoing FIGGS survey (e.g., Begum et al. 2008) will likely fill this gap.

The results presented here confirm earlier findings by $\mathrm{CvG}$ and Broeils (1992) that the rotation curve shape depends on morphology and luminosity (at least for galaxies brighter than approximately $M_{R}=-19 \mathrm{mag}$ ). However, with the inclusion of the galaxies from the sample presented here, and a larger number of spiral galaxies from the sample of Verheijen \& Sancisi (2001), the correlation between rotation curve shape and morphology and luminosity is better defined, especially at the low luminosity end. Based on these new data, we find that the outer rotation curves of galaxies with Hubble types later than Sc or with absolute magnitudes fainter than approximately $M_{R}=-19 \mathrm{mag}$ all have similar shapes. In other words, we find that the outer rotation curve shape does not depend on luminosity or morphology for galaxies fainter than $M_{R} \sim-19$ for the sample presented here.

The main reason for this difference in the reported correlation between rotation curve shape on the one hand and Hubble type or absolute magnitude on the other lies in the derived rotation curve shapes for the late-type dwarf galaxies presented here compared to those derived from rotation curves as published in the literature. Both beam smearing (see Sect. 3 and references therein) and intensity-weighted velocities (see Paper I and references therein) will lead to a shallower derived rotation curves, especially where the rotation curve is rising or turning over. These differences may well explain the differences in derived rotation curves.

Considering the bright galaxies that have outer rotationcurve slopes different from those of late-type dwarf galaxies, it is interesting to note that the vast majority of these have a bulge (or a strong central concentration of light). These bulges have a strong effect on the shape of the inner rotation shape, causing a steep rise in the center. But the effect of these bulges may extend to larger radii as well and explain the falling rotation curves seen in some galaxies (e.g. Noordermeer et al. 2007). Given that we found that the outer rotation curve shapes for galaxies fainter than $M_{R} \sim-19$ (which overwhelmingly do not have bulges) do not depend on luminosity, it is tempting to speculate that the change in rotation curve shapes for brighter galaxies is the result from the increasing contribution of the bulge, while the rotation curve shape of the underlying disk remains unchanged.

We have found a relation between the shape of the central distribution of light and the inner rise of the rotation curve. Galaxies with a stronger central concentration of light have a more steeply rising rotation curve. This correlation between the light distribution and the inner rise of the rotation curve is well known for spiral galaxies (e.g., Kent 1987; Corradi \& Capaccioli 1990), but in this paper we have found that dwarf galaxies with a central concentration of light also have rotation curves that rise more steeply in the center than the rotation curves of dwarf galaxies that do not have a central concentration of light.

The observed correlation between the light distribution and the inner rotation curve shape, as seen in both spiral and late-type dwarf galaxies, implies that galaxies with stronger central concentrations of light also have higher central mass densities, and it suggests that the luminous mass dominates the gravitational potential in the central regions, even in low surface brightness dwarf galaxies.

Acknowledgements. We are grateful to Martin Vogelaar, Hans Terlouw and Kor Begeman for the implementation of the programs used here in the GIPSY software package. We thank Marc Verheijen and Erwin de Blok for making their data available in electronic format. This research has made use of the NASA/IPAC Extragalactic Database (NED) which is operated by the Jet Propulsion Laboratory, California Institute of Technology, under contract with the National Aeronautics and Space Administration.

\section{Appendix A: Rotation curves}

In the next pages overviews are presented of the rotation curves for all galaxies in this study. For each galaxy a figure is given with two panels. In the left panel, the position-velocity diagram 
along the major axis is shown. Contour levels are at $-4 \sigma$ and $-2 \sigma$ (dotted), and $2 \sigma, 4 \sigma, \ldots$ Overlayed on the position-velocity diagram is the rotation curve. If the rotation curve has been derived for the approaching and the receding side separately, then these are shown. In the right panel, the derived rotation curve (represented by the thick solid line and the filled circles) is shown. If the rotation curves for the approaching and receding sides were derived separately, both are shown, together with the average. The thin solid line represents the the approaching side, the dotted line the receding side. The arrow at the bottom of the panel indicates a radius of 2 optical disk scale lengths.

\section{References}

Begeman, K. 1987, Ph.D. Thesis, Rijksuniversiteit Groningen Begeman, K. G. 1989, A\&A, 223, 47

Begum, A., Chengalur, J. N., Karachentsev, I. D., Sharina, M. E., \& Kaisin, S. S. 2008, MNRAS, 386, 1667

Binney, J., \& Tremaine, S. 1987, Galactic Dynamics (Princeton University Press) Blais-Ouellette, S., Amram, P., \& Carignan, C. 2001, AJ, 121, 1952

Bosma, A. 1978, Ph.D. Thesis, Rijksuniversiteit Groningen

Bosma, A. 1981a, AJ, 86, 1791

Bosma, A. 1981b, AJ, 86, 1825

Bottema, R., Shostak, G. S., \& van der Kruit, P. C. 1986, A\&A, 167, 34

Broeils, A. H. 1992a, Ph.D. Thesis, Rijksuniversiteit Groningen

Broeils, A. H. 1992b, A\&A, 256, 19

Bullock, J. S., Kolatt, T. S., Sigad, Y., et al. 2001, MNRAS, 321, 559

Casertano, S., \& van Gorkom, J. H. 1991, AJ, 101, 1231 (CvG)

Catinella, B., Giovanelli, R., \& Haynes, M. P. 2006, ApJ, 640, 751

Chengalur, J. N., Begum, A., Karachentsev, I. D., Sharina, M., \& Kaisin, S. S. 2008, in Galaxies in the Local Volume, ed. B. Koribalski, \& H. Jerjen, in print, see also [arXiv: 0711.2153]

Corradi, R. L. M., \& Capaccioli, M. 1990, A\&A, 237, 36

Côté, S., Carignan, C., \& Freeman, K. C. 2000, AJ, 120, 3027

de Blok, W. J. G., \& Bosma, A. 2002, A\&A, 385, 816 (dBB)

de Blok, W. J. G., \& McGaugh, S. S. 1997, MNRAS, 290, 533

de Blok, W. J. G., McGaugh, S. S., \& van der Hulst, J. M. 1996, MNRAS, 283, 18

de Blok, W. J. G., McGaugh, S. S., \& Rubin, V. C. 2001, AJ, 122, 2396

de Vaucouleurs, G., de Vaucouleurs, A., Corwin, H. G., et al. 1991, Third

Reference Catalogue of Bright Galaxies (New York: Springer)

García-Ruiz, I., Sancisi, R., \& Kuijken, K. 2002, A\&A, 394, 769

Garrido, O., Marcelin, M., Amram, P., \& Boulesteix, J. 2002, A\&A, 387, 821

Hunter, D. A., Rubin, V. C., Swaters, R. A., Sparke, L. S., \& Levine, S. E. 2002, ApJ, 580, 194
Kent, S. M. 1986, AJ, 91, 1301

Kent, S. M. 1987, AJ, 93, 816

Kregel, M., \& van der Kruit, P. C. 2004, MNRAS, 352, 787

Kuzio de Naray, R., McGaugh, S. S., de Blok, W. J. G., \& Bosma, A. 2006, ApJS, 165, 461

Marchesini, D., D'Onghia, E., Chincarini, G., et al. 2002, ApJ, 575, 801

McGaugh, S. S., Rubin, V. C., \& de Blok, W. J. G. 2001, AJ, 122, 2381

Navarro, J. F., Frenk, C. S., \& White, S. D. M. 1996, ApJ, 462, 563

Navarro, J. F., Frenk, C. S., \& White, S. D. M. 1997, ApJ, 490, 493

Nilson, P. 1973, Uppsala General Catalogue of Galaxies, Uppsala Astr. Obs. Ann., 6 (UGC)

Noordermeer, E., van der Hulst, J. M., Sancisi, R., Swaters, R. S., \& van Albada, T. S. 2007, MNRAS, 376, 1513

Persic, M., \& Salucci, P. 1988, MNRAS, 234, 131

Persic, M., Salucci, P., \& Stel, F. 1996, MNRAS, 281, 27

Power, C., Navarro, J. F., Jenkins, A., et al. 2003, MNRAS, 338, 14

Puche, D., Westpfahl, D., Brinks, E., \& Roy, J.-R. 1992, AJ, 103, 1841

Rhee, M.-Y. 1996, Ph.D. Thesis, Rijksuniversiteit Groningen

Rhee, G., Valenzuela, O., Klypin, A., Holtzman, J., \& Moorthy, B. 2004, ApJ, 617,1059

Rubin, V. C., Burstein, D., Ford, Jr., W. K., \& Thonnard, N. 1985, ApJ, 289, 81

Rubin, V. C., Kenney, J. D., Boss, A. P., \& Ford, W. K. 1989, AJ, 98, 1246

Sancisi, R., \& Allen, R. J. 1979, A\&A, 74, 73

Sicotte, V., \& Carignan, C. 1997, AJ, 113, 609

Sicotte, V., Carignan, C., Durand, D. 1996, AJ 112, 1423

Simon, J. D., Bolatto, A. D., Leroy, A., Blitz, L., \& Gates, E. L. 2005, ApJ, 621, 757

Spekkens, K., \& Giovanelli, R. 2006, AJ, 132, 1426

Spekkens, K., Giovanelli, R., \& Haynes, M. P. 2005, AJ, 129, 2119

Stil, J. M. 1999, Ph.D. Thesis, University of Leiden

Swaters, R. A. 1999, Ph.D. Thesis, Rijksuniversiteit Groningen

Swaters, R. A., \& Balcells, M. 2002, A\&A, 390, 863 (Paper II)

Swaters, R. A., Sancisi, R., \& van der Hulst, J. M. 1997, ApJ, 491, 140

Swaters, R. A., Schoenmakers, R. H. M., Sancisi, R., \& van Albada, T. S. 1999, MNRAS, 304, 330

Swaters, R. A., Madore, B. F., \& Trewhella, M. 2000, ApJ, 531, L107

Swaters, R. A., van Albada, T. S., van der Hulst, J. M., \& Sancisi, R. 2002, A\&A, 390, 829 (Paper I)

Swaters, R. A., Madore, B. F., van den Bosch, F. C., \& Balcells, M. 2003a, ApJ, 583, 732 (SMvdBB)

Swaters, R. A., Verheijen, M. A. W., Bershady, M. A., \& Andersen, D. R. 2003b, ApJ, 587, L19

Tully, R. B., Bottinelli, L., Fisher, J. R., et al. 1978, A\&A, 63, 37

van den Bosch, F. C., \& Swaters, R. A. 2001, MNRAS, 325, 1017

van den Bosch, F. C., Robertson, B. E., Dalcanton, J. J., \& de Blok, W. J. G. 2000, AJ, 119, 1579

Verheijen, M. A. W. 1997, Ph.D. Thesis, Rijksuniversiteit Groningen

Verheijen, M. A. W., \& Sancisi, R. 2001, A\&A, 370, 765 

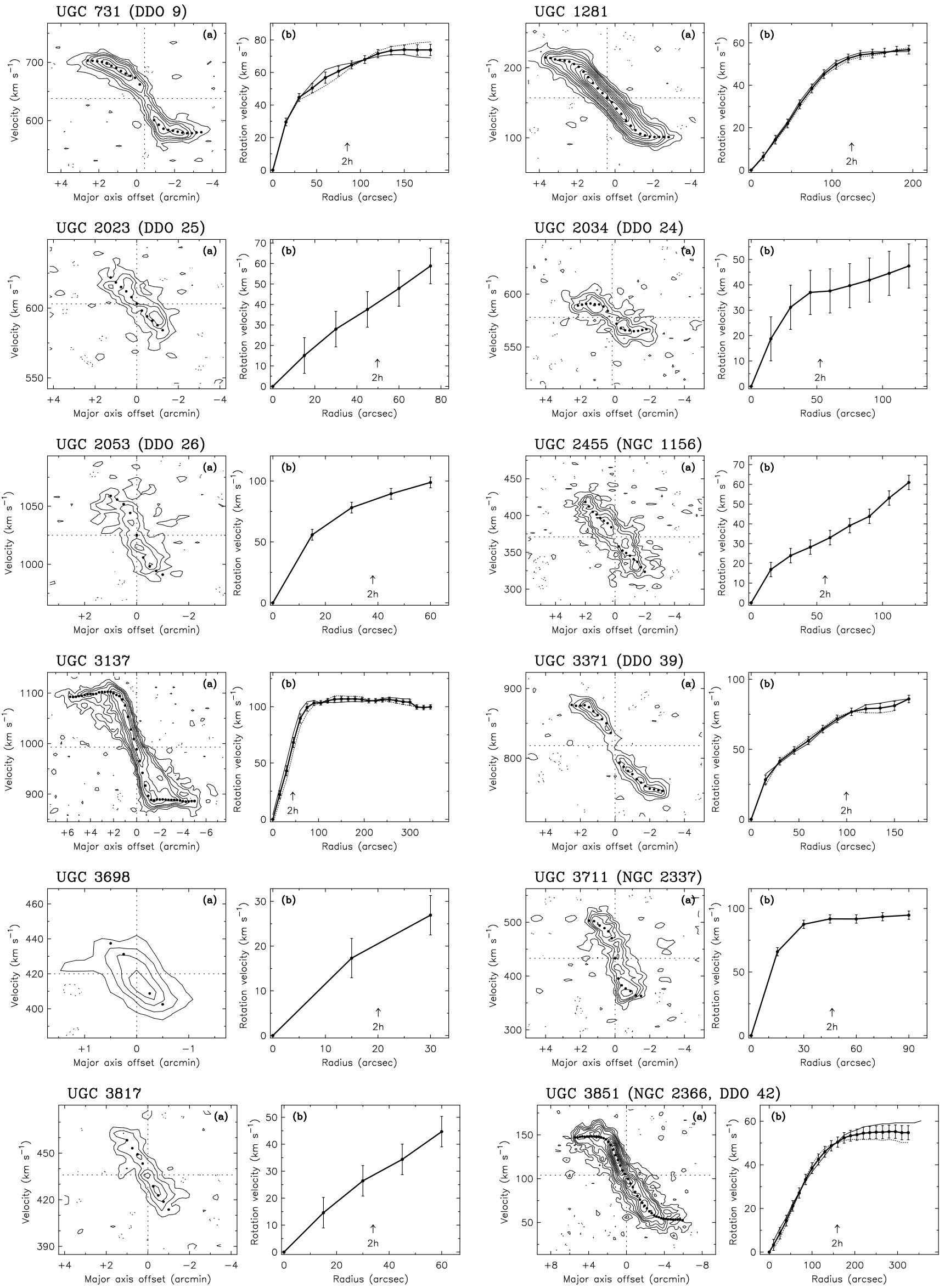

Fig. A.1. 

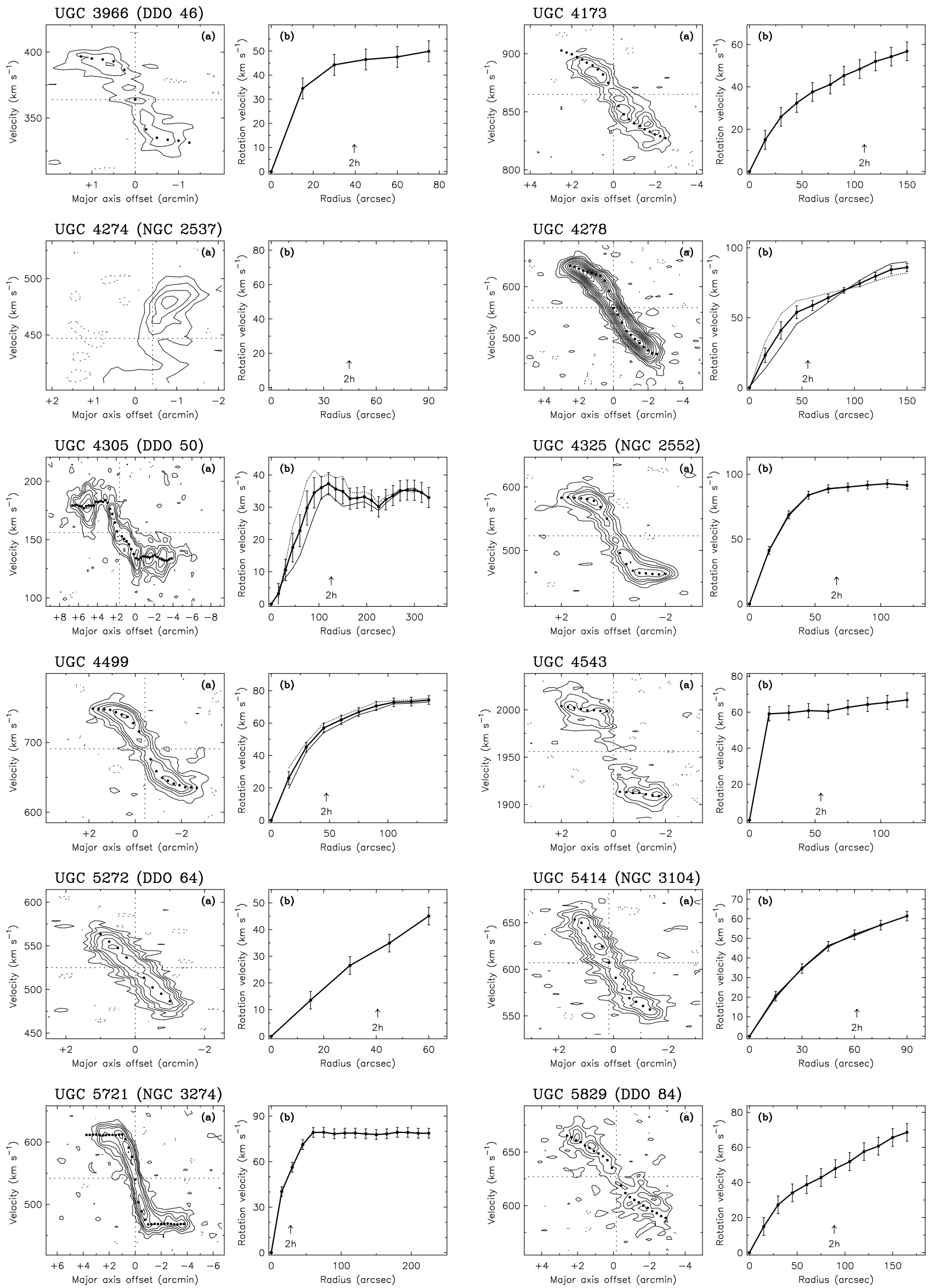

Fig. A.1. continued. 

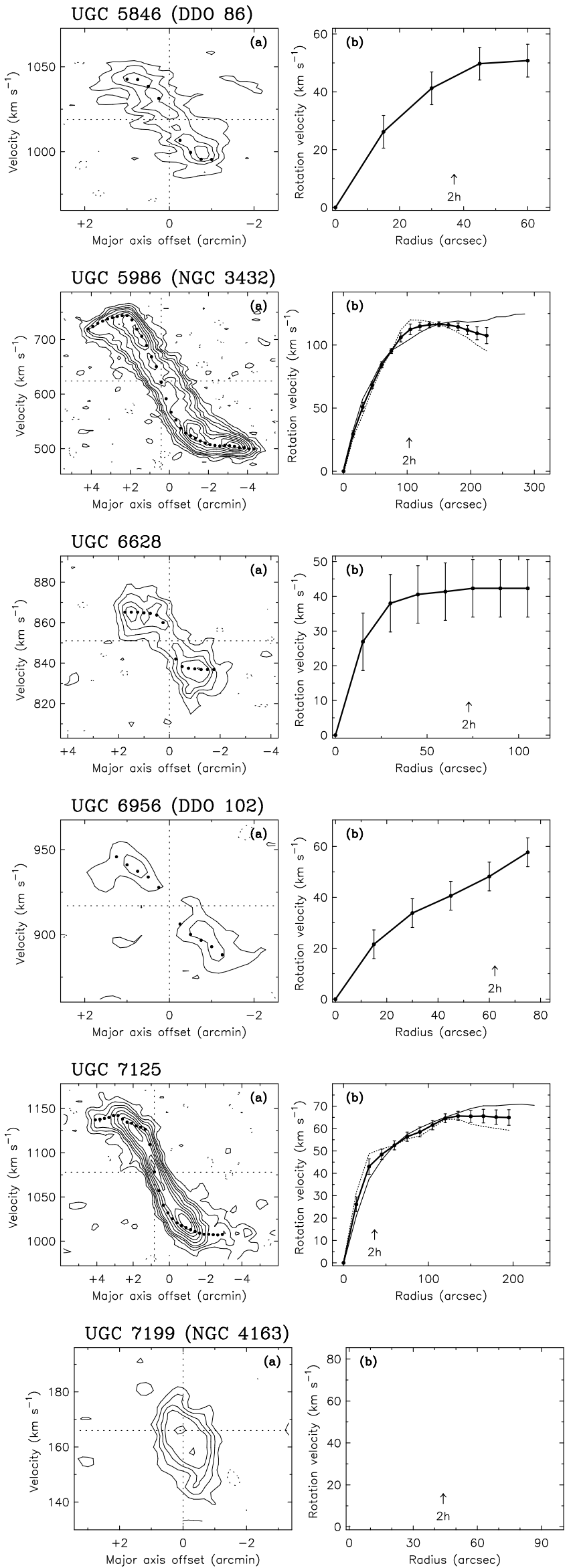
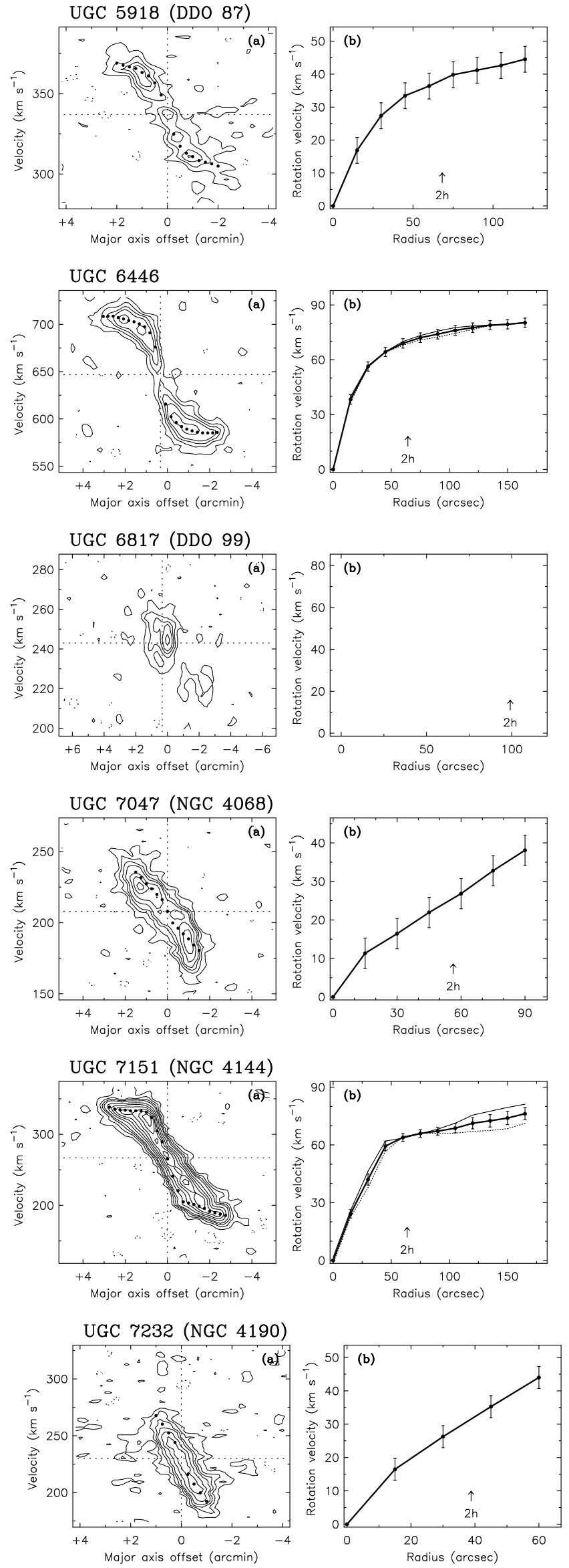

Fig. A.1. continued. 

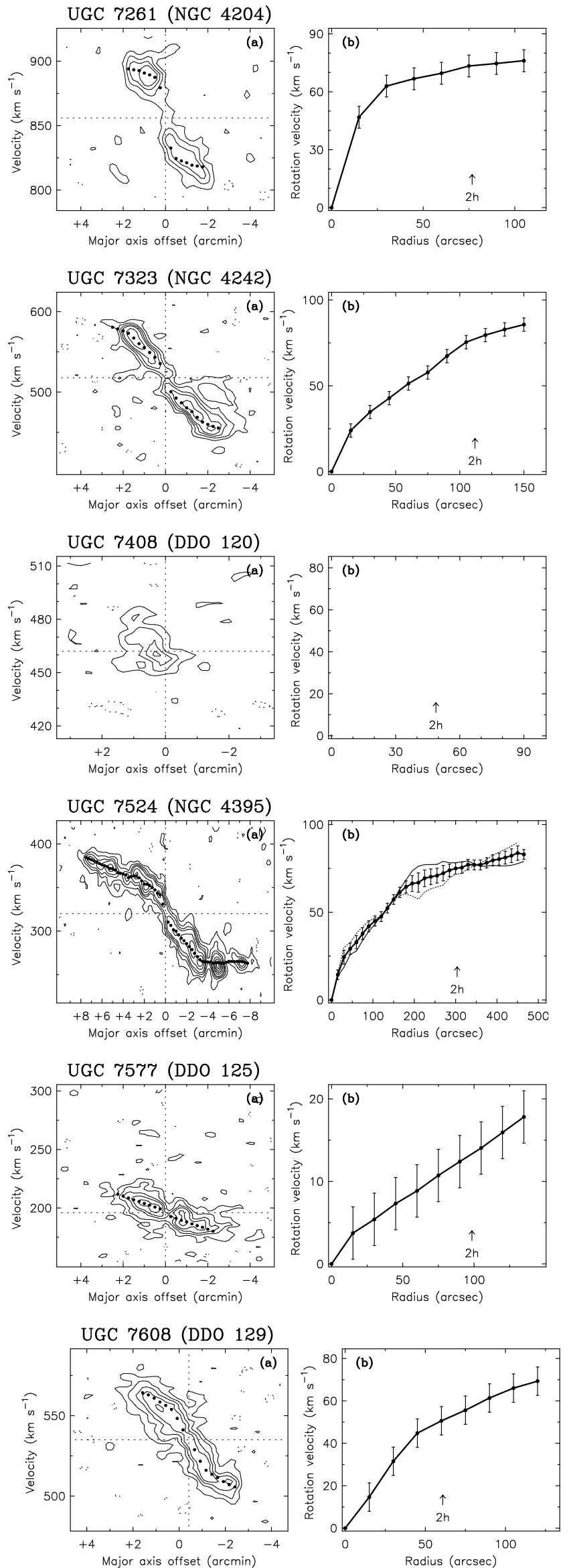

UGC 7278 (NGC 4214)
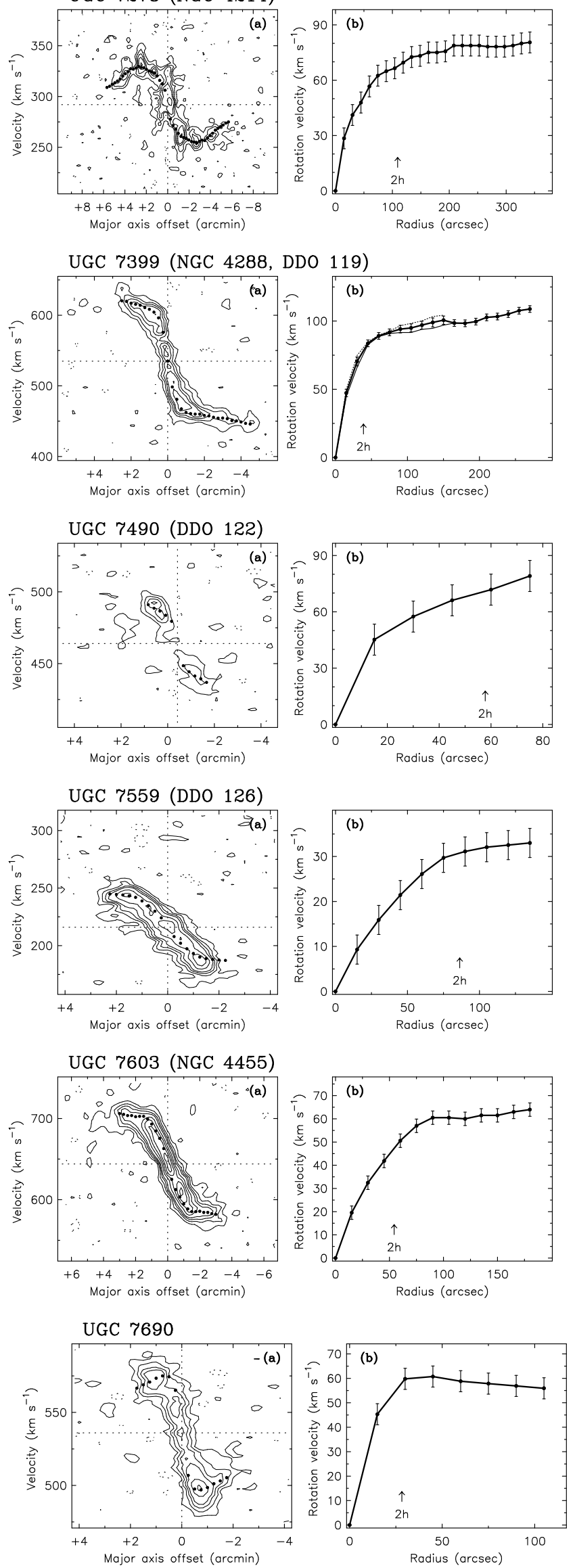

Fig. A.1. continued. 
R. A. Swaters et al.: The rotation curves shapes of late-type dwarf galaxies

UGC 7866 (DDO 141)
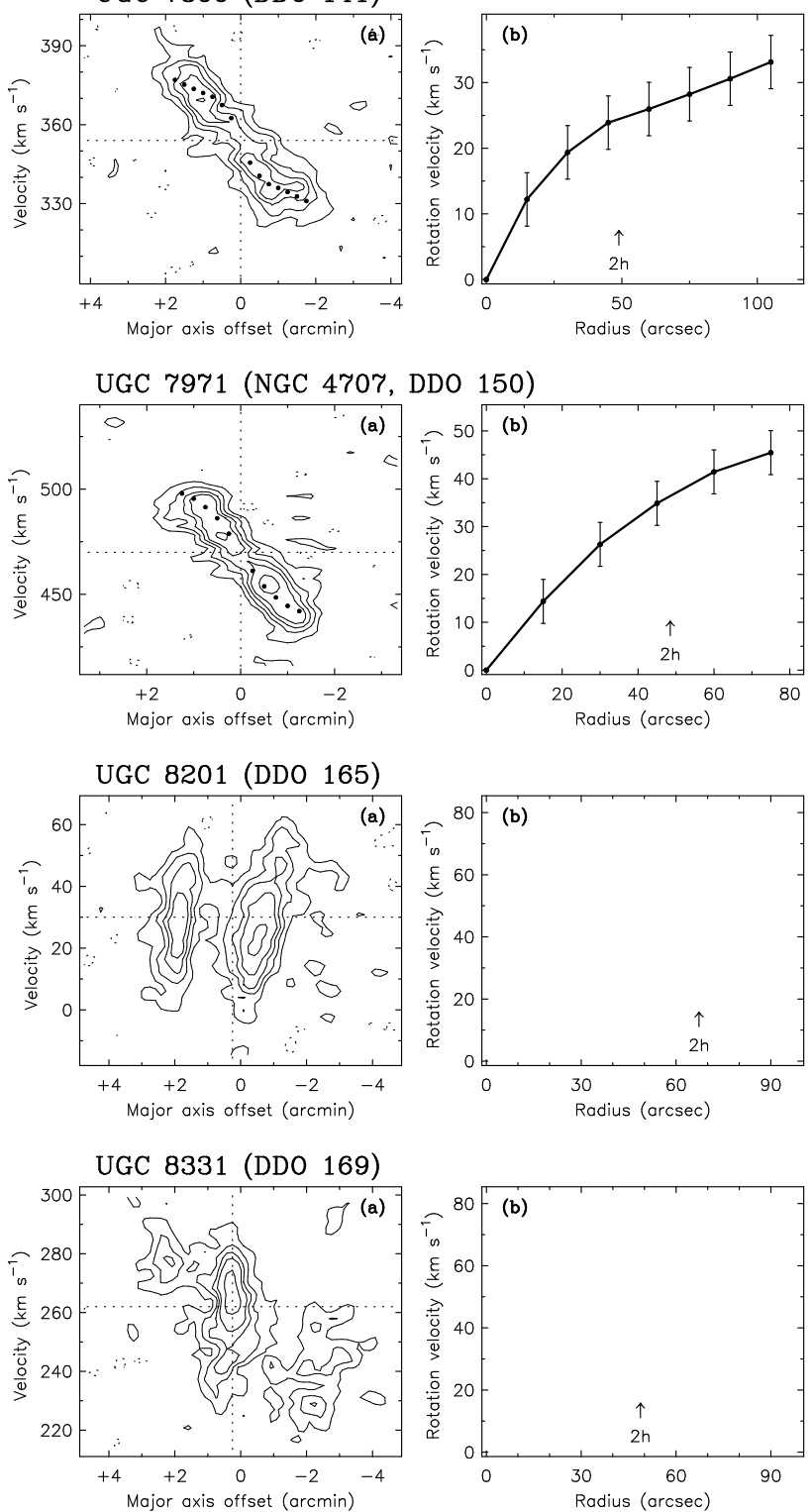

UGC 8550 (NGC 5229)
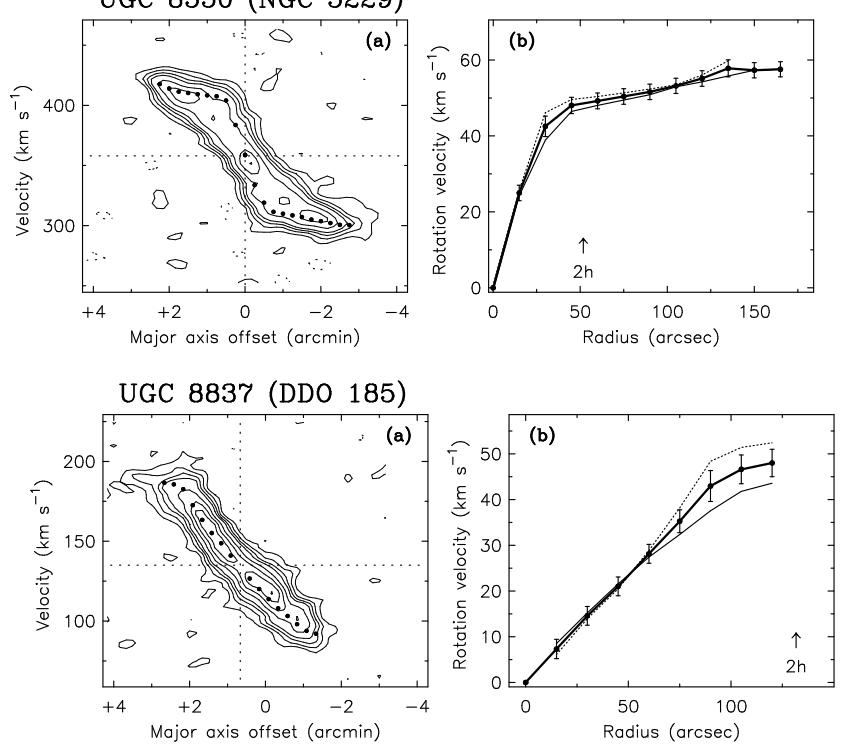
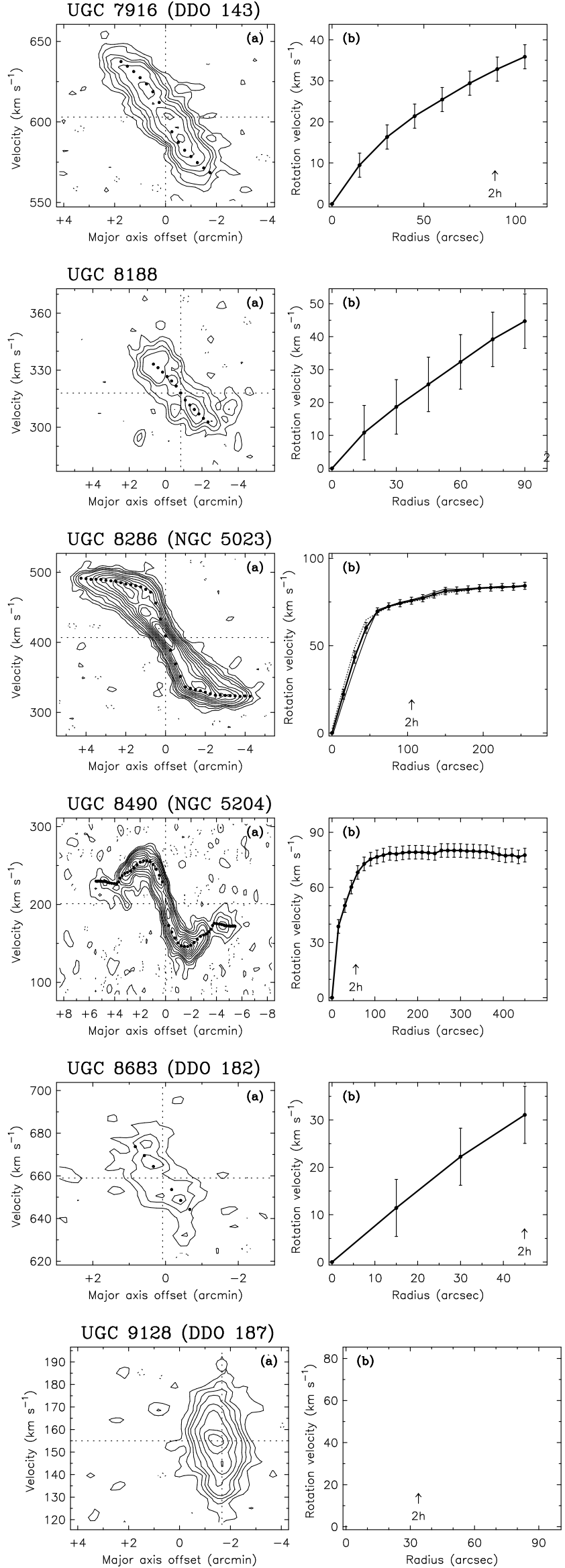

Fig. A.1. continued. 

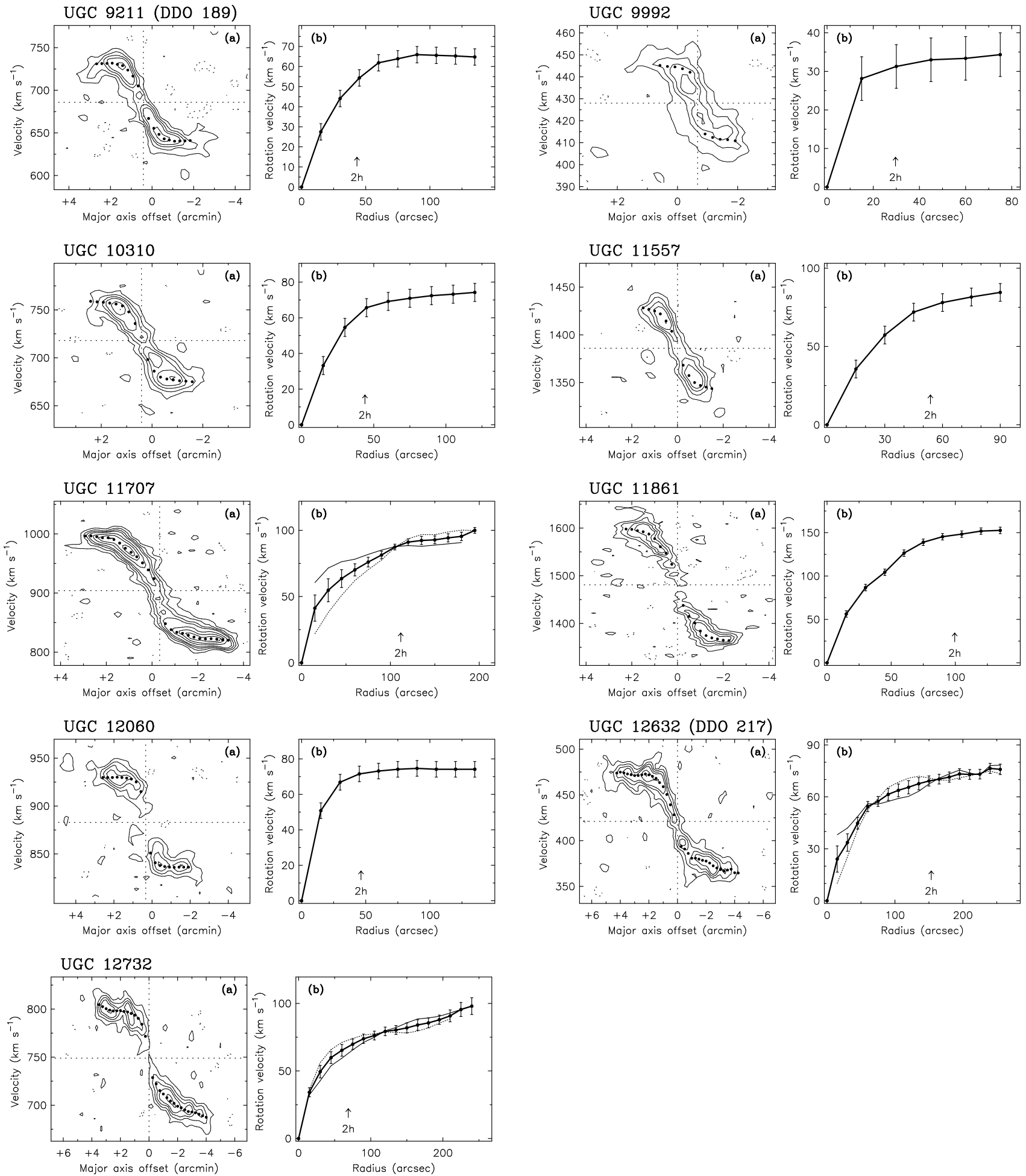

Fig. A.1. continued. 